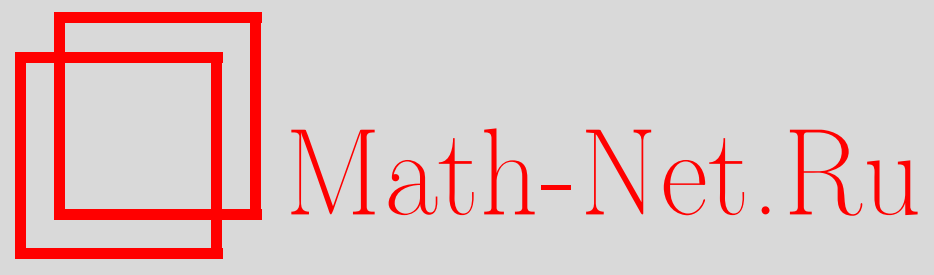

В. А. Ватутин, Е. Е. Дьяконова, Предельные теоремы для редуцированных ветвящихся процессов в случайной среде, Теория вероятн. и ее примен., 2007, том 52, выпуск 2, 271-300

DOI: https://doi.org/10.4213/tvp173

Использование Общероссийского математического портала Math-Net.Ru подразумевает, что вы прочитали и согласны с пользовательским соглашением

http: //www . mathnet.ru/rus/agreement

Параметры загрузки:

IP : 18.209 .158 .208

26 апреля 2023 г., 11:23:03

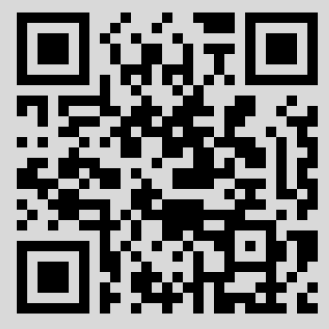




\section{ПРЕДЕЛЬНЫЕ ТЕОРЕМЫ ДЛЯ РЕДУЦИРОВАННЫХ ВЕТВЯЩИХСЯ ПРОЦЕССОВ В СЛУЧАЙНОЙ СРЕДЕ}

Рассматривается ветвящийся процесс $Z(n), n=0,1, \ldots$, в случайной среде, порожденной последовательностью независимых одинаково распределенных производящих функций $f_{0}(s), f_{1}(s), \ldots$ Пусть $S_{0}=0, S_{k}=X_{1}+\cdots+X_{k}, k \geqslant 1,-$ сопровождающее случайное блуждание этого процесса, $X_{i}=\ln f_{i-1}^{\prime}(1)$, а $\tau(n)$ - самая левая точка, в которой достигается минимум блуждания $\left\{S_{k}\right\}_{k \geqslant 0}$ на интервале $[0, n]$. Обозначим $Z(k, n)$ число частиц, существовавших в ветвящемся процессе в момент времени $k \leqslant n$ и имеющих ненулевое потомство в момент времени $n$. В предположении, что для сопровождающего случайного блуждания выполнено условие СпицераДони $\mathbf{P}\left\{S_{n}>0\right\} \rightarrow \rho \in(0,1), n \rightarrow \infty$, показано (в рамках так называемого quenched approach), что для любого фиксированного $m=0, \pm 1, \pm 2, \ldots$ распределение случайной величины $Z(\tau(n)+m, n)$ при условии $Z(n)>0$ сходится при $n \rightarrow \infty$ к (случайному) дискретному распределению, являюшемуся собственным с вероятностью 1 . В случае же, когда $m=m(n) \rightarrow \infty$ при $n \rightarrow \infty$, для доказательства аналогичной условной предельной теоремы о распределении случайной величины $Z(\tau(n)+m, n)$ при условии $Z(n)>0$ необходимо нормировать $Z(\tau(n)+m, n)$ некоторой функцией, стремящейся к бесконечности с ростом $m$.

Ключевые слова и фразы: ветвящиеся процессы в случайной среде, условие Спицера-Дони, условные предельные теоремы, редуцированные процессы, ближайший общий предок.

Введение. Настоящая статья продолжает работы [7]-[11], посвященные изучению таких ветвящихся процессов в случайной среде, в которых вероятностные законы размножения частиц различных поколений независимы и одинаково распределены.

Неформальное описание рассматриваемой нами модели ветвящихся процессов выглядит следующим образом. Пусть $\mathbf{F}=\{f(s)\}$ - множество всех производяцих функций, соответствующих вероятностным

* Математический институт им. В. А. Стеклова РАН, ул. Губкина, 8, 119991 Москва, ГСП-1, Россия; е-mail: vatutin@mi.ras.ru, elena@mi.ras.ru

1) Работа выполнена при финансовой поддержке РФФИ (грант 05-01-00035), программы поддержки ведуших научных школ НШ-4129.2006.1, INTAS (проект 03-51-5018), ECO-NET 12634TJ и программы РАН «Современные проблемы теоретической математики». 
распределениям с носителями, сосредоточенными на множестве неотрицательных целых чисел $\mathbf{N}_{0}$, снабженное равномерной метрикой. Предположим, что на борелевских подмножествах пространства $\mathbf{F}$ задана вероятностная мера $\mathbb{P}$. Выбирая (с возвращением) из $\mathbf{F}$ в соответствии с указанной мерой последовательность производящих функций $f_{0}(s)$, $f_{1}(s), \ldots, f_{n}(s), \ldots$ и фиксируя ее, мы получаем бесконечную последовательность называемую (случайной) средой. Эта среда порождает неоднородный ветвящийся процесс $\{Z(n), n \geqslant 0\}$, задаваемый выражениями

$$
\begin{aligned}
& Z(0)=1, \\
& \mathbf{E}\left[s^{Z(n+1)} \mid f_{0}, f_{1}, \ldots, f_{n} ; Z(0), Z(1), \ldots, Z(n)\right]=\left(f_{n}(s)\right)^{Z(n)}, \quad n \geqslant 0,
\end{aligned}
$$

где

$$
f_{n, \pi}(s)=f_{n}(s):=\sum_{k=0}^{\infty} \pi_{n}(k) s^{k} .
$$

Полученная таким образом последовательность $\{Z(n), n \geqslant 0\}$ называется ветвящимся процессом в случайной среде. Мы ограничимся лишь этим описанием интересующей нас модели ветвящихся процессов в случайной среде. Более строгое описание и построение таких процессов можно найти в [1]-[3].

Иногда нам будет удобнее отождествлять вероятностную производящую функцию $f_{n}(s)$ с бесконечномерным вектором $\boldsymbol{\pi}_{n}$ :

$$
\boldsymbol{\pi}_{n}=\left\{\pi_{n}(0), \pi_{n}(1), \pi_{n}(2), \ldots\right\}, \quad \pi_{n}(k) \geqslant 0, \quad \sum_{k=0}^{\infty} \pi_{n}(k)=1, \quad n \in \mathbf{N}_{0}
$$

Пусть $f \stackrel{d}{=} f_{0}, X_{j}:=\ln f_{j-1}^{\prime}(1), \eta_{j}:=f_{j-1}^{\prime \prime}(1)\left(f_{j-1}^{\prime}(1)\right)^{-2}, j \in \mathbf{N}=$ $\{1,2, \ldots\} ; X \stackrel{d}{=} X_{1}, S_{0}:=0, S_{n}:=X_{1}+\cdots+X_{n}, n \geqslant 1$. Мы будем называть последовательность $\left\{S_{n}\right\}_{n \geqslant 0}$ сопровождающим случайным блужданием ветвящегося процесса в случайной среде.

Скажем, что ветвящийся процесс в случайной среде является невырожденным критическим, если $\limsup _{n \rightarrow \infty} S_{n}=+\infty$ и $\liminf _{n \rightarrow \infty} S_{n}=$ $-\infty$ с вероятностью 1 , и вырожденным критическим, если $S_{n}=0, n \geqslant 0$ (более подробное обоснование этой терминологии см. в [4] и [11]). В дальнейшем мы рассматриваем исключительно невырожденные критические ветвящиеся процессы в случайной среде и для краткости называем их просто критическими ветвящимися процессами в случайной среде, опуская слово «невырожденные».

Пусть $\tau\left(n_{1}, n_{2}\right):=\min \left\{i \in\left[n_{1}, n_{2}\right]: S_{j} \geqslant S_{i}, j=n_{1}, n_{1}+1, \ldots, n_{2}\right\}-$ самая левая точка интервала $\left[n_{1}, n_{2}\right]$, в которой достигается минимальное значение случайного блуждания $\left\{S_{n}\right\}_{n \geqslant 0}$. Для краткости положим $\tau(n):=\tau(0, n)$. В работах [10] и [11] (в рамках так называемого quenched approach, т.е. в ситуации, когда вероятности событий 
и меры рассматриваются как случайные элементы, зависящие от реализации среды) был доказан ряд условных предельных теорем, описывающих асимптотическое поведение распределения числа частиц в критическом ветвящемся процессе в случайной среде при условии невырождения процесса к моменту $n \rightarrow \infty$. В частности, было показано, что если $t \in(0,1]$ фиксировано, то (случайное) условное распределение случайной величины $Z([n t]) e^{S_{\tau([n t])}-S_{[n t]}}$ при условии $Z(n)>0$ сходится в некотором смысле к собственному предельному распределению, не имеющему атома в нуле, в то время как при фиксированном $t \in(0,1]$ и $\oint$ фисированном $m \in \mathbf{Z}=\{0, \pm 1, \pm 2, \ldots\}$ (случайное) распределение величины $Z(\tau([n t])+m)$ при условии $Z(n)>0$ сходится (в некотором смысле) к (случайному) собственному дискретному предельному распределению.

В настоящей работе мы рассматриваем еще один важный процесс, построенный по ветвящемуся процессу $\{Z(k), 0 \leqslant k \leqslant n\}$, а именно, мы изучаем так называемый редуцированный ветвящийся процесс $\{Z(k, n)$, $0 \leqslant k \leqslant n\}$, где $Z(k, n)$ - число частиц в первоначальном процессе в момент времени $k \leqslant n$, имеющих ненулевое число потомков в момент времени $n$. Редуцированные процессы для обычных процессов ГальтонаВатсона впервые рассмотрели Фляйшманн и Прен [18], которые исследовали докритический случай. Критические и надкритические редуцированные процессы Гальтона-Ватсона изучали Зубков [15] и Фляйшманн и Зигмунд-Шультце [19]. Первые результаты для редуцированных ветвящихся процессов в случайной среде получили Боровков и Ватутин [5], а также Фляйшманн и Ватутин [17]. Применяя так называемый annealed approach (т.е. усредняя характеристики и меры относительно распределения $\mathbb{P}$, заданного на пространстве сред), они рассматривали случай, в котором носитель меры $\mathbb{P}$ сконцентрирован лишь на множестве дробнолинейных производящих функций. В paмках annealed approach Ватутин [6] доказал предельную теорему для критических редуцированных процессов в случайной среде для производящих функций общего вида.

В данной работе мы рассматриваем quenched approach и развиваем методы, предложенные в [7]-[11]. Мы покажем, что при условии $Z(n)>0$ конечномерные условные (случайные) распределения процесса $\left\{Z([n t], n) e^{S_{\tau([n t], n)}-S_{\tau(n)}}, 0 \leqslant t \leqslant 1\right\}$ сходятся (в некотором смысле) к конечномерным распределениям некоторого предельного процесса, а конечномерные условные (случайные) распределения процесса $\{Z(\tau(n)+m, n)$, $m \in \mathbf{Z}\}$ сходятся (в некотором смысле) к конечномерным распределениям ветвящегося процесса Гальтона-Ватсона в неоднородной случайной среде.

1. Основные условия. Введем сначала основные условия, которые впоследствии всегда будут считаться выполненными. Так, мы будем предполагать, что распределение случайной величины $X$ либо не- 
решетчато, либо центрально решетчато (т.е. $X$ принимает лишь значения $h k, h>0, k \in \mathbf{Z}$, и $\mathbf{P}\{X=0\}>0)$ и что сопровождающее случайное блуждание удовлетворяет следующему условию условию СпицераДони.

Условие $A 1$. Существует число $0<\rho<1$ такое, что при $n \rightarrow \infty$

$$
\frac{1}{n} \sum_{k=1}^{n} \mathbf{P}\left\{S_{k}>0\right\} \rightarrow \rho
$$

или, что то же самое (см. [13]), $\mathbf{P}\left\{S_{n}>0\right\} \rightarrow \rho, n \rightarrow \infty$.

Известно, что при выполнении условия $A 1$

$$
n^{-1} \tau(n) \stackrel{w}{\longrightarrow} \tau, \quad n \rightarrow \infty,
$$

где $\tau$ - случайная величина, распределенная по обобщенному закону арксинуса с параметром $\rho$ (см. $[16$, гл. IV, $\S 20])$, а символ $\stackrel{w}{\longrightarrow}$ означает слабую сходимость.

Пусть $\gamma_{0}:=0, \gamma_{j+1}:=\min \left(n>\gamma_{j}: S_{n}<S_{\gamma_{j}}\right)$ и $\Gamma_{0}:=0, \Gamma_{j+1}:=$ $\min \left(n>\Gamma_{j}: S_{n}>S_{\Gamma_{j}}\right), j \geqslant 0$, - строгие убывающие и, соответственно, строгие возрастающие лестничные моменты случайного блуждания $\left\{S_{n}\right\}_{n \geqslant 0}$. Введем функции

$$
\begin{aligned}
& V(x):=\sum_{j=0}^{\infty} \mathbf{P}\left\{S_{\gamma_{j}} \geqslant-x\right\}, \quad x>0, \quad V(0)=1, \quad V(x)=0, \quad x<0, \\
& U(x):=1+\sum_{j=1}^{\infty} \mathbf{P}\left\{S_{\Gamma_{j}}<x\right\}, \quad x>0, \quad U(0)=1, \quad U(x)=0, \quad x<0,
\end{aligned}
$$

и положим

$$
\Theta(a):=\sum_{k=a}^{\infty} k^{2} \pi(k) /\left(\sum_{k=0}^{\infty} k \pi(k)\right)^{2}, \quad a \in \mathbf{N}_{0} .
$$

Условие $A 2$. Существуют числа $\varepsilon_{0}>0$ и $a \in \mathbf{N}_{0}$ такие, что

$$
\begin{array}{rlll}
\mathbf{E}\left(\ln ^{+} \Theta(a)\right)^{1 / \rho+\varepsilon_{0}}<\infty & \text { и } & \mathbf{E}\left[V(X)\left(\ln ^{+} \Theta(a)\right)^{1+\varepsilon_{0}}\right]<\infty, \\
\mathbf{E}\left(\ln ^{+} \Theta(a)\right)^{1 /(1-\rho)+\varepsilon_{0}}<\infty & \text { и } & \mathbf{E}\left[U(-X)\left(\ln ^{+} \Theta(a)\right)^{1+\varepsilon_{0}}\right]<\infty .
\end{array}
$$

Более детальное обсуждение этих условий можно найти в [4] и [10].

Пусть $\Omega_{n}$ - пространство элементарных событий, соответствуюшее наборам $f_{0}, f_{1}, \ldots, f_{n-1}, Z(0), Z(1), \ldots, Z(n) ; \Omega=\Omega_{\infty}$, а

$$
\mathscr{F}_{n}=\sigma\left(f_{0}, f_{1}, \ldots, f_{n-1} ; Z(0), Z(1), \ldots, Z(n)\right), \quad n \in \mathbf{N}_{0}, \quad \mathscr{F}:=\bigvee_{n} \mathscr{F}_{n}
$$

- последовательность естественных $\sigma$-алгебр, порожденных ветвяшимся процессом в случайной среде. В дальнейшем нам потребуются различные вероятностные меры, определенные на измеримом пространстве $(\Omega, \mathscr{F})$. Чтобы отличать их друг от друга, мы будем использовать символы $\mathbf{E}$ и $\mathbf{P}$ для обозначения математического ожидания и вероятности относительно вероятностной 
меры, порождаемой исходной мерой ветвяшегося процесса на набо$\operatorname{pax}\left(f_{0}, f_{1}, \ldots, f_{n-1}, \ldots ; Z(0), Z(1), \ldots, Z(n), \ldots\right)$ (за исключением некоторых случаев, которые, как мы надеемся, не приведут к недоразумениям), символы $\mathbb{E}$ и $\mathbb{P}$ используются для обозначения математического ожидания и вероятности относительно маргинальной меры на множестве сред, а символы $\mathbf{E}_{\pi}, \mathbf{P}_{\pi}$ - для обозначения условного математического ожидания и условной вероятности при фиксированной среде $\boldsymbol{\pi}=\left(\boldsymbol{\pi}_{0}, \boldsymbol{\pi}_{1}, \ldots, \boldsymbol{\pi}_{n}, \ldots\right)$. Мы будем использовать в рассуждениях как пространство $(\Omega, \mathscr{F}, \mathbf{P})$, так и две его копии $\left(\Omega^{-}, \mathscr{F}^{-}, \mathbf{P}^{-}\right)$ и $\left(\Omega^{+}, \mathscr{F}^{+}, \mathbf{P}^{+}\right)$. Рассмотрим две последовательности случайных элементов $\left\{f_{n}^{-}\right\}_{n \geqslant 0}$ и $\left\{f_{n}^{+}\right\}_{n \geqslant 0}$, заданных на пространствах $\left(\Omega^{-}, \mathscr{F}^{-}, \mathbf{P}^{-}\right)$ и $\left(\Omega^{+}, \mathscr{F}^{+}, \mathbf{P}^{+}\right)$, соответственно, и обозначим $\left\{S_{n}^{-}\right\}_{n \geqslant 0}$ и $\left\{S_{n}^{+}\right\}_{n \geqslant 0}$ порожденные ими сопровождающие случайные блуждания, которые, естественно, определены на соответствующих пространствах $\left(\Omega^{-}, \mathscr{F}^{-}, \mathbf{P}^{-}\right)$ и $\left(\Omega^{+}, \mathscr{F}^{+}, \mathbf{P}^{+}\right)$. В дальнейшем все характеристики или случайные величины, связанные с $\left\{f_{n}^{-}\right\}_{n \geqslant 0}$ и $\left\{f_{n}^{+}\right\}_{n \geqslant 0}$, будут снабжаться индексами и + , соответственно. Например, мы будем использовать случайные величины $\Gamma^{-}:=\min \left\{n \geqslant 1: S_{n}^{-} \geqslant 0\right\}$ и $\gamma^{+}:=\min \left\{n \geqslant 1: S_{n}^{+}<0\right\}$. Кроме того, мы будем изучать различные свойства пары ветвящихся процессов в случайной среде, заданных на $\left(\Omega^{-} \times \Omega^{+}, \mathscr{F}^{-} \times \mathscr{F}^{+}, \mathbf{P}^{-} \times \mathbf{P}^{+}\right)$, при условии осуществления события $\mathscr{A}_{k, p}:=\left\{\Gamma^{-}>k, \gamma^{+}>p\right\}$.

Положим $D=\sum_{j=1}^{\infty} j^{-1} \mathbf{P}\left\{S_{j}=0\right\}$. Наряду с исходными мерами $\mathbf{P}^{-}$ и $\mathbf{P}^{+}$, определим на $\left(\Omega^{-}, \mathscr{F}^{-}\right)$и $\left(\Omega^{+}, \mathscr{F}^{+}\right)$меры $\widehat{\mathbf{P}}^{-}$и $\widehat{\mathbf{P}}^{+}$, полагая для $\mathscr{A}^{-} \in \mathscr{F}_{k}^{-}, \mathscr{A}^{+} \in \mathscr{F}_{p}^{+}, k, p \in \mathbf{N}$

$$
\begin{aligned}
& \widehat{\mathbf{P}}^{-}\left(\mathscr{A}^{-}\right):=e^{D} \int_{\mathscr{A}^{-}} U\left(-S_{k}^{-}\right) I\left\{\Gamma^{-}>k\right\} d \mathbf{P}^{-} \\
& \widehat{\mathbf{P}}^{+}\left(\mathscr{A}^{+}\right):=\int_{\mathscr{A}^{+}} V\left(S_{p}^{+}\right) I\left\{\gamma^{+}>p\right\} d \mathbf{P}^{+}
\end{aligned}
$$

где $I\{\mathscr{A}\}$ - индикатор события $\mathscr{A}$. Нетрудно понять вероятностный смысл этих мер: носитель сужения меры $\widehat{\mathbf{P}}^{-}$на $\mathscr{F}_{k}^{-}$сконцентрирован лишь на тех реализациях случайной среды, сопровождающие случайные блуждания которых остаются отрицательными в течении первых $k$ шагов (за исключением начальной точки), в то время как носитель сужения меры $\widehat{\mathbf{P}}^{+}$на $\mathscr{F}_{k}^{+}$сконцентрирован только на реализациях среды, сопровождающие случайные блуждания которых в течение первых $k$ шагов остаются неотрицательными.

На измеримом пространстве $\left(\Omega^{-} \times \Omega^{+}, \mathscr{F}^{-} \times \mathscr{F}^{+}\right)$зададим вероятностную меру $\widehat{\mathbf{P}}:=\widehat{\mathbf{P}}^{-} \times \widehat{\mathbf{P}}^{+}$, полагая для $\mathscr{A} \in \mathscr{F}_{k}^{-} \times \mathscr{F}_{p}^{+}$

$$
\widehat{\mathbf{P}}(\mathscr{A}):=e^{D} \int_{\mathscr{A}} U\left(-S_{k}^{-}\right) V\left(S_{p}^{+}\right) I\left\{\mathscr{A}_{k, p}\right\} d\left(\mathbf{P}^{-} \times \mathbf{P}^{+}\right)
$$

(более подробное описание этих мер можно найти в [11]). 
Ниже мы будем использовать символы $\widehat{\mathbf{E}}^{ \pm}, \widehat{\mathbf{E}}$ и $\mathscr{L}^{ \pm}, \mathscr{L}$ для обозначения математических ожиданий и законов распределения относительно мер $\widehat{\mathbf{P}}^{ \pm}$и $\widehat{\mathbf{P}}$, соответственно.

Пусть $\mathbf{M}=\mathbf{M}([0, \infty))$ - множество всех (возможно, несобственных) вероятностных мер на $[0, \infty)$, снабженное топологией слабой сходимости, $\mathbf{M}_{+}-$подмножество мер $\mathscr{M}$ в $\mathbf{M}$ с $\mathscr{M}(\{0\})=0$. Обозначим $\mathbf{m}=\mathbf{m}(\{0,1, \ldots\})$ множество всех мер $\mu \in \mathbf{M}$ с носителями, принадлежацими множеству $\{0,1, \ldots\}$, и положим $\mathbf{m}_{+}=\mathbf{m} \cap \mathbf{M}_{+}$. С каждой мерой $\mu \in \mathbf{m}$ мы свяжем соответствующую ей производящую функцию

$$
h(s):=\sum_{k=0}^{\infty} \mu(k) s^{k}
$$

и для последовательности производящих функций

$$
h^{(n)}(s)=\sum_{k=0}^{\infty} \mu^{(n)}(k) s^{k}, \quad n \in \mathbf{N}_{0}
$$

(здесь и далее верхний индекс используется для обозначения номера серии, а не порядка производной) будем писать $h^{(n)}(s) \stackrel{w}{\longrightarrow} h(s), s \in[0,1)$, при $n \rightarrow \infty$, если $\mu^{(n)} \stackrel{w}{\longrightarrow} \mu, n \rightarrow \infty$.

Статья построена следующим образом. В разделе 2 мы исследуем структуру редуцированного критического ветвящегося процесса в случайной среде в моменты времени, близкие к точке глобального минимума сопровождающего случайного блуждания, и устанавливаем сходимость конечномерных распределений процесса $\{Z(k, n), 0 \leqslant k \leqslant n\}$ к конечномерным распределениям некоторого неоднородного ветвящегося процесса. В частности, мы покажем, что ближайший общий предок всех частиц, существующих в процессе в момент времени $n$, расположен «неподалеку» от момента $\tau(n)$ глобального минимума сопровождающего случайного блуждания на интервале $[0, n]$. В разделе 3 мы изучим структуру редуцированного критического процесса в случайной среде в моменты времени, находящиеся значительно правее точки $\tau(n)$. Наконец, в разделах 4 и 5 мы применим результаты, полученные в разделах 2 и 3 , к исследованию структуры процесса $\{Z([n t], n), 0 \leqslant t \leqslant 1\}$.

\section{2. Поведение редуцированного процесса вблизи точки гло-} бального минимума сопровождающего случайного блуждания. В этом разделе мы изучим (в рамках quenched approach) структуру редуцированного процесса $\{Z(k, n), 0 \leqslant k \leqslant n\}$ в случайной среде вблизи момента $\tau(n)$ глобального минимума сопровождающего случайного блуждания на отрезке $[0, n]$ при $n \rightarrow \infty$. В качестве следствия наших результатов мы найдем распределение расстояния до ближайшего общего предка всех частиц, существующих в процессе в момент времени $n$. 
Положим

$$
\begin{aligned}
f_{k, n}(s):=f_{k}\left(f_{k+1}\left(\ldots\left(f_{n-1}(s)\right) \ldots\right)\right), \quad 0 \leqslant k \leqslant n-1, \quad f_{n, n}(s):=s \\
f_{n, m}(s):=f_{n-1}\left(f_{n-2}\left(\ldots\left(f_{m}(s)\right) \ldots\right)\right), \quad n \geqslant m+1 .
\end{aligned}
$$

Ниже мы приводим несколько результатов, установленных в [10], на которые будем ссылаться в дальнейшем, предполагая при этом, что пара $\left\{f_{n}^{-}, f_{n}^{+}\right\}_{n \geqslant 0}$ задана на $\left(\Omega^{-} \times \Omega^{+}, \mathscr{F}^{-} \times \mathscr{F}^{+}\right)$и что ей соответствует пара сопровождающих случайных блужданий $\left\{S_{n}^{-}, S_{n}^{+}\right\}_{n \geqslant 0}$. Сформулируем необходимые нам утверждения.

Если выполнены условия $A 1-A 2$, то для любого $m \in \mathbf{N}_{0}$ существует предел

$$
q_{m}^{+}:=\lim _{n \rightarrow \infty} f_{m, n}^{+}(0)<1
$$

$\widehat{\mathbf{P}}$-п.н. (далее мы для краткости вместо $q_{0}^{+}$будем писать $q^{+}$); более того, если положить

$$
\zeta_{l, m}^{-}(s):=\frac{1-f_{l, m}^{-}(s)}{e^{S_{l}^{-}-S_{m}^{-}}}, \quad m \in \mathbf{N}_{0}, \quad l \geqslant m+1,
$$

то $\widehat{\mathbf{P}}$-п.н. существует и является положительным предел

$$
\zeta_{\infty, m}^{-}(s):=\lim _{l \rightarrow \infty} \zeta_{l, m}^{-}(s) \leqslant 1
$$

причем

$$
\lim _{s \uparrow 1} \zeta_{\infty, m}^{-}(s)=0 .
$$

Для краткости положим $\zeta_{l}^{-}(s):=\zeta_{l, 0}^{-}(s)$ и $\zeta^{-}(s):=\zeta_{\infty, 0}^{-}(s)$. Заметим, что согласно (2)-(4) $\widehat{\mathbf{P}}$-п.н. предел

$$
\zeta:=\lim _{\min (l, r) \rightarrow \infty} \zeta_{l}^{-}\left(f_{0, r}^{+}(0)\right)=\zeta^{-}\left(q^{+}\right)
$$

существует, положителен и не превосходит единицы. Пусть

$$
\begin{aligned}
h_{m, \pi}^{(n)}(s)=h_{m}^{(n)}(s) & :=\mathbf{E}_{\pi}\left[s^{Z(\tau(n)+m, n)} \mid Z(n)>0\right] \\
& :=\sum_{k=1}^{\infty} \mu_{m}^{(n)}(k) s^{k}, \quad m \in \mathbf{Z} .
\end{aligned}
$$

Для $m \geqslant 0$ положим

$$
\begin{gathered}
h_{m}(s):=1-\frac{\zeta^{-}\left(f_{0, m}^{+}\left(1-(1-s)\left(1-q_{m}^{+}\right)\right)\right)}{\zeta} \\
h_{-m}(s):=1-\frac{\zeta_{\infty, m}^{-}\left(1-(1-s)\left(1-f_{m, 0}^{-}\left(q^{+}\right)\right)\right)}{e^{S_{m}^{-}} \zeta} .
\end{gathered}
$$

Нам будет удобно записывать $h_{m}(s), m \in \mathbf{Z}$, в виде

$$
h_{m}(s):=\sum_{k=1}^{\infty} \mu_{m}(k) s^{k} .
$$


Ниже мы будем трактовать $\mu_{m}^{(n)}$ и $\mu_{m}$ как случайные меры, распределения которых на $\mathbf{m}$ индуцируются последовательностями сред, выбранных согласно вероятностным мерам $\mathbf{P}$ и $\widehat{\mathbf{P}}$, соответственно. Для этих случайных объектов будем обозначать через $\mu_{m}^{(n)} \stackrel{w(\mathbf{P}, \widehat{\mathbf{P}})}{\longmapsto} \mu_{m}, n \rightarrow \infty$, следующий вид слабой сходимости случайных мер $\mu_{m}^{(n)}$ к $\mu_{m}$ при $n \rightarrow \infty$ : для любой неслучайной ограниченной функции $J: \mathbf{N}_{0} \rightarrow \mathbf{R}$ и любой неслучайной непрерывной функции $g: \mathbf{R} \rightarrow \mathbf{R}$ с компактным носителем

$$
\mathbf{E}\left[g\left(\mu_{m}^{(n)}[J]\right)\right] \longrightarrow \widehat{\mathbf{E}}\left[g\left(\mu_{m}[J]\right)\right], \quad n \rightarrow \infty .
$$

Здесь для меры $\mu \in \mathbf{m}$ и функции $J: \mathbf{N}_{0} \rightarrow \mathbf{R}$ мы полагаем $\mu[J]:=$ $\sum_{k \geqslant 0} J(k) \mu(k)$.

Иногда нам будет удобнее записывать (10) в терминах соответствующих производящих функций в виде $h_{m}^{(n)}(s) \stackrel{w(\mathbf{P}, \widehat{\mathbf{P}})}{\longmapsto} h_{m}(s), s \in[0,1)$, $n \rightarrow \infty$. Эти же обозначения будут использоваться и в других аналогичных ситуациях.

Теперь мы можем сформулировать условную предельную теорему (в рамках quenched approach) о распределении числа частиц в редуцированном процессе в моменты времени, расположенные вблизи точки глобального минимума сопровождающего случайного блуждания.

Теорема 1. Если выполнены условия $A 1-A 2$, то для любого $m \in \mathbf{Z}$

$$
\mu_{m}^{(n)} \stackrel{w(\mathbf{P}, \widehat{\mathbf{P}})}{\longmapsto} \mu_{m}, \quad n \rightarrow \infty
$$

или, что то же самое,

$$
h_{m}^{(n)}(s) \stackrel{w(\mathbf{P}, \widehat{\mathbf{P}})}{\longmapsto} h_{m}(s), \quad s \in[0,1), \quad n \rightarrow \infty .
$$

Д о к аз а т е л ь с т в о. Нетрудно проверить, что для $m \geqslant 0$

$$
h_{m}^{(n)}(s)=1-\frac{1-f_{0, \tau(n)}\left(f_{\tau(n), \tau(n)+m}\left(1-(1-s)\left(1-f_{\tau(n)+m, n}(0)\right)\right)\right)}{1-f_{0, \tau(n)}\left(f_{\tau(n), n}(0)\right)} .
$$

Ясно, что

$$
\mathbf{E}\left[h_{m}^{(n)}(s) \mid \tau(n)=l\right]=\mathbf{E}\left[h_{m}^{(l, n-l)}(s) \mid \mathscr{A}_{l, n-l}\right]
$$

где

$$
\begin{aligned}
h_{m}^{(l, n-l)}(s) & :=1-\frac{1-f_{l, 0}^{-}\left(f_{0, m}^{+}\left(1-(1-s)\left(1-f_{m, n-l}^{+}(0)\right)\right)\right)}{1-f_{l, 0}^{-}\left(f_{0, n-l}^{+}(0)\right)} \\
& =1-\frac{\zeta_{l}^{-}\left(f_{0, m}^{+}\left(1-(1-s)\left(1-f_{m, n-l}^{+}(0)\right)\right)\right)}{\zeta_{l}^{-}\left(f_{0, n-l}^{+}(0)\right)} \\
& =: \sum_{k=1}^{\infty} \mu_{m}^{(l, n-l)}(k) s^{k}
\end{aligned}
$$


а $\left(\left(f_{n}^{-}\right)_{n \geqslant 0},\left(f_{n}^{+}\right)_{n \geqslant 0}\right)$ - пара независимых последовательностей независимых одинаково распределенных производящих функций, заданных на $\left(\Omega^{-} \times \Omega^{+}, \mathscr{F}^{-} \times \mathscr{F}^{+}, \mathbf{P}^{-} \times \mathbf{P}^{+}\right)$. Фактически, равенство (13) означает, что для любого множества $\mathscr{B} \subseteq \mathbf{N}$

$$
\left\{\mu_{m}^{(n)}(\mathscr{B}) \mid \tau(n)=l\right\} \stackrel{d}{=}\left\{\mu_{m}^{(l, n-l)}(\mathscr{B}) \mid \mathscr{A}_{l, n-l}\right\}
$$

Как мы знаем из $(3)-(6),(7)$ и $(9), \widehat{\mathbf{P}}$-п.н. существует предел

$$
\lim _{\min (l, n-l) \rightarrow \infty} h_{m}^{(l, n-l)}(s)=1-\frac{\zeta^{-}\left(f_{0, m}^{+}\left(1-(1-s)\left(1-q_{m}^{+}\right)\right)\right)}{\zeta}=h_{m}(s)
$$

или, что то же самое, $\widehat{\mathbf{P}}$-п.н. $\mu_{m}^{(l, n-l)} \stackrel{w}{\longrightarrow} \mu_{m}$ при $\min (l, n-l) \rightarrow \infty$. Следовательно, для любой неслучайной ограниченной функции $J: \mathbf{N} \rightarrow \mathbf{R}$ и любой неслучайной непрерывной функции $g: \mathbf{R} \rightarrow \mathbf{R}$ с компактным носителем

$$
\lim _{\min (l, n-l) \rightarrow \infty} g\left(\mu_{m}^{(l, n-l)}[J]\right)=g\left(\mu_{m}[J]\right) \quad \widehat{\mathbf{P}} \text {-п.н. }
$$

Для того, чтобы вывести отсюда (12) и (11) для $m \in \mathbf{N}_{0}$, достаточно заметить, что ввиду (14)

$$
\mathbf{E}\left[g\left(\mu_{m}^{(n)}[J]\right) \mid \tau(n)=l\right]=\left(\mathbf{E}^{-} \times \mathbf{E}^{+}\right)\left[g\left(\mu_{m}^{(l, n-l)}[J]\right) \mid \mathscr{A}_{l, n-l}\right],
$$

и применить лемму 4 из [10].

Аналогично, для $m>0$

$$
h_{-m}^{(n)}(s)=1-\frac{1-f_{0, \tau(n)-m}\left(1-(1-s)\left(1-f_{\tau(n)-m, \tau(n)}\left(f_{\tau(n), n}(0)\right)\right)\right)}{1-f_{0, n}(0)}
$$

и соотношение (13) по-прежнему справедливо, где теперь

$$
\begin{aligned}
h_{-m}^{(l, n-l)}(s) & :=1-\frac{1-f_{l, m}^{-}\left(1-(1-s)\left(1-f_{m, 0}^{-}\left(f_{0, n-l}^{+}(0)\right)\right)\right)}{1-f_{l, 0}^{-}\left(f_{0, n-l}^{+}(0)\right)} \\
& =1-\frac{\zeta_{l, m}^{-}\left(1-(1-s)\left(1-f_{m, 0}^{-}\left(f_{0, n-l}^{+}(0)\right)\right)\right)}{\zeta_{l}^{-}\left(f_{0, n-l}^{+}(0)\right)} .
\end{aligned}
$$

Ввиду (3)-(6) и (8) $\widehat{\mathbf{P}}$-п.н. существует предел

$$
\lim _{\min (l, n-l) \rightarrow \infty} h_{-m}^{(l, n-l)}(s)=1-\frac{\zeta_{\infty, m}^{-}\left(1-(1-s)\left(1-f_{m, 0}^{-}\left(q^{+}\right)\right)\right)}{e^{S_{m}^{-}} \zeta}=h_{-m}(s)
$$

что и завершает доказательство соотношения (11) для всех $m$.

Теорема 1 доказана.

Из теоремы 1 вытекает следующее интересное следствие о распределении случайной величины $B(n)=\max \{0 \leqslant k \leqslant n: Z(k, n)=1\}$, называемой моментом рождения ближайшего общего предка всех частиц, существующих в популяции в момент времени $n$. 
Следствие 1. Если выполнень условия $A 1-A 2$, то для любого $m \in \mathbf{N}_{0}$

$$
\begin{aligned}
R_{-m} & :=\lim _{n \rightarrow \infty} \mathbf{P}_{\pi}(B(n) \geqslant \tau(n)-m)=\lim _{n \rightarrow \infty} \mathbf{P}_{\pi}(Z(\tau(n)-m, n)=1) \\
& \stackrel{d}{=} h_{-m}^{\prime}(0)=\left.\left(\zeta_{\infty, m}^{-}(s)\right)^{\prime}\right|_{s=f_{m, 0}^{-}\left(q^{+}\right)}\left(1-f_{m, 0}^{-}\left(q^{+}\right)\right)
\end{aligned}
$$

$u$

$$
\begin{aligned}
R_{m} & :=\lim _{n \rightarrow \infty} \mathbf{P}_{\pi}(B(n) \geqslant \tau(n)+m)=\lim _{n \rightarrow \infty} \mathbf{P}_{\pi}(Z(\tau(n)+m, n)=1) \\
& \stackrel{d}{=} h_{m}^{\prime}(0)=\left.\left(\zeta^{-}\left(f_{m, 0}^{+}(s)\right)\right)^{\prime}\right|_{s=q_{m}^{+}}\left(1-q_{m}^{+}\right) .
\end{aligned}
$$

Более того, с вероятностью 1

$$
\begin{gathered}
\lim _{m \rightarrow \infty} R_{-m}=\lim _{m \rightarrow \infty} \lim _{n \rightarrow \infty} \mathbf{P}_{\pi}(Z(\tau(n)-m, n)=1)=1, \\
\lim _{m \rightarrow \infty} R_{m}=\lim _{m \rightarrow \infty} \lim _{n \rightarrow \infty} \mathbf{P}_{\pi}(Z(\tau(n)+m, n)=1)=0 .
\end{gathered}
$$

Д о к а з а т е л ь с т в о. Соотношения (15) и (16) несложно получить из теоремы 1 прямыми вычислениями.

Для доказательства (17) и (18) достаточно показать, что $\widehat{\mathbf{P}}$-п.н.

$$
\lim _{m \rightarrow \infty} h_{-m}(s)=s \quad \text { и } \quad \lim _{m \rightarrow \infty} h_{m}(s)=0 .
$$

Чтобы проверить первое из этих предельных соотношений, заметим, что согласно (3)-(6)

$$
\begin{aligned}
& e^{-S_{m}^{-}} \zeta_{\infty, m}^{-}\left(1-(1-s)\left(1-f_{m, 0}^{-}\left(q^{+}\right)\right)\right) \leqslant(1-s) e^{-S_{m}^{-}}\left(1-f_{m, 0}^{-}\left(q^{+}\right)\right) \\
& \underset{m \rightarrow \infty}{\longrightarrow}(1-s) \zeta \hat{\mathbf{P}} \text {-п.н. }
\end{aligned}
$$

Следовательно, $\widehat{\mathbf{P}}$-п.н.

$$
\liminf _{m \rightarrow \infty} h_{-m}(s)=\liminf _{m \rightarrow \infty}\left[1-\frac{\zeta_{\infty, m}^{-}\left(s\left(1-f_{m, 0}^{-}\left(q^{+}\right)\right)+f_{m, 0}^{-}\left(q^{+}\right)\right)}{e^{S_{m}^{-}} \zeta}\right] \geqslant s .
$$

С другой стороны, ввиду (5) для $m>0$

$$
h_{-m}(1)=1-\frac{\zeta_{\infty, m}^{-}(1)}{e^{S_{m}^{-}} \zeta}=1 \quad \widehat{\mathbf{P}} \text {-п.н. }
$$

и из $(2)-(6)$ имеем

$$
\begin{aligned}
h_{-m}(0) & =1-\frac{\zeta_{\infty, m}^{-}\left(f_{m, 0}^{-}\left(q^{+}\right)\right)}{e^{S_{m}^{-}} \zeta}=1-\frac{1}{e^{S_{m}^{-}} \zeta} \lim _{l \rightarrow \infty} \frac{1-f_{l, m}^{-}\left(f_{m, 0}^{-}\left(q^{+}\right)\right)}{e^{S_{l}^{-}-S_{m}^{-}}} \\
& =1-\frac{1}{\zeta} \lim _{l \rightarrow \infty} \frac{1-f_{l, 0}^{-}\left(q^{+}\right)}{e^{S_{l}^{-}}}=0 \quad \widehat{\mathbf{P}} \text {-п.н. }
\end{aligned}
$$

Таким образом, для $m>0 \widehat{\mathbf{P}}$-п.н.

$$
h_{-m}(s)=\sum_{k=1}^{\infty} \mu_{-m}(k) s^{k} \leqslant s
$$

Отсюда и из (20) следует первое из соотношений в (19). 
Для доказательства второго заметим, что в соответствии с (2) $q_{m}^{+}<1 \widehat{\mathbf{P}}^{+}$-п.н., и, кроме того, в силу предложения 3.1 в [4] (см., в частности, формулу (3.6)) имеет место соотношение

$$
\sum_{i=0}^{\infty}\left(1-\left(f_{i}^{+}\right)^{\prime}(0)\right)=\infty \quad \widehat{\mathbf{P}}^{+} \text {-п.н. }
$$

Отсюда, согласно теореме 5 работы [1], следует, что для всех $s \in[0,1)$

$$
\lim _{m \rightarrow \infty} f_{0, m}^{+}(s)=\lim _{m \rightarrow \infty} f_{0, m}^{+}(0)=q^{+} \quad \widehat{\mathbf{P}}_{\text {-п.н. }}
$$

и, таким образом, $\widehat{\mathbf{P}}$-п.н.

$$
\begin{aligned}
\lim _{m \rightarrow \infty} h_{m}(s) & =\lim _{m \rightarrow \infty}\left[1-\frac{\zeta^{-}\left(f_{0, m}^{+}\left(1-(1-s)\left(1-q_{m}^{+}\right)\right)\right)}{\zeta}\right] \\
& =1-\frac{\zeta^{-}\left(\lim _{m \rightarrow \infty} f_{0, m}^{+}\left(1-(1-s)\left(1-q_{m}^{+}\right)\right)\right)}{\zeta} \\
& =1-\frac{\zeta^{-}\left(q^{+}\right)}{\zeta}=0 .
\end{aligned}
$$

Следствие доказано.

3 а м е ч а н и е 1 . Следствие 1 допускает следуюшую неформальную интерпретацию. Если популяция выжила к моменту времени $n \rightarrow \infty$, то момент рождения ближайшего общего предка всех частиц, существующих в момент времени $n$, расположен в окрестности точки $\tau(n)$. Так как при выполнении условия $A 1 \tau(n) n^{-1}$ сходится по распределению при $n \rightarrow \infty$ к случайной величине, распределенной согласно обобщенному закону арксинуса на интервале $[0,1]$, то в рассматриваемом нами случае полученные результаты разительно отличаются от соответствующих результатов для обычных критических ветвяцихся процессов Гальтона-Ватсона, в которых расстояние до ближайшего общего предка, нормированное на $n$, имеет равномерное распределение на интервале $[0,1]$ (см., например, [19]).

Рассмотрим теперь распределение редуцированного процесса в окрестности точки $\tau(n)$ более детально. Для этого введем следующие обозначения:

$$
\begin{aligned}
w_{k, n, \pi}(s) & =w_{k, n}(s):=\mathbf{E}_{\pi}\left[s^{Z(k+1, n)} \mid Z(n)>0, Z(k)=1\right] \\
& =\mathbf{E}_{\pi}\left[s^{Z(k+1, n)} \mid Z(k, n)=1\right], \quad 0 \leqslant k<n,
\end{aligned}
$$

и $w_{n, n}(s):=1$. Нетрудно показать, что

$$
\begin{aligned}
w_{k, n}(s) & =\frac{f_{k}\left(f_{k+1, n}(0)+s\left(1-f_{k+1, n}(0)\right)\right)-f_{k, n}(0)}{1-f_{k, n}(0)} \\
& =1-\frac{1-f_{k}\left(1-(1-s)\left(1-f_{k+1, n}(0)\right)\right)}{1-f_{k, n}(0)}
\end{aligned}
$$


Для $0 \leqslant \tau(n)+m \leqslant n$ положим

$$
a_{m}^{(n)}(s):=w_{\tau(n)+m, n}(s)=\mathbf{E}_{\pi}\left[s^{Z(\tau(n)+m+1, n)} \mid Z(\tau(n)+m, n)=1\right]
$$

и для $m \in \mathbf{N}_{0}$ обозначим

$$
\begin{aligned}
a_{-m}(s) & :=\frac{f_{m}^{-}\left(f_{m-1,0}^{-}\left(q^{+}\right)+s\left(1-f_{m-1,0}^{-}\left(q^{+}\right)\right)\right)-f_{m, 0}^{-}\left(q^{+}\right)}{1-f_{m, 0}^{-}\left(q^{+}\right)} \\
a_{m}(s) & :=\frac{f_{m}^{+}\left(q_{m+1}^{+}+s\left(1-q_{m+1}^{+}\right)\right)-q_{m}^{+}}{1-q_{m}^{+}} .
\end{aligned}
$$

Следующая теорема описывает распределение числа частиц в редуцированном процессе, построенном по ветвящемуся процессу, порожденному одной частицей, родившейся в момент времени, расположенный вблизи точки глобального минимума сопровождающего случайного блуждания.

Теорема 2. Если выполнены условия $A 1-A 2$, то для любого $m \in \mathbf{Z}$

$$
a_{m}^{(n)}(s) \stackrel{w(\mathbf{P}, \widehat{\mathbf{P}})}{\longmapsto} a_{m}(s), \quad s \in[0,1), \quad n \rightarrow \infty .
$$

Д о к а з а т е л ь с т в о. Для доказательства этой теоремы к соотношениям (23) и (22) нужно применить (с незначительными изменениями) рассуждения, проводившиеся при доказательстве теоремы 1. Детали необходимых аргументов мы опускаем.

П р и м е р. Нетрудно показать, используя формулу (2.2) в [12], что если выполнены условия $A 1-A 2$ и мера $\mathbb{P}$ сконцентрирована на вероятностных производящих функциях геометрических распределений, т.е. на функциях, имеющих вид $f(s)=q(1-p s)^{-1}, p+q=1, p q>0$, то $\widehat{\mathbf{P}}$-п.н.

$$
\begin{aligned}
\zeta_{\infty, m}^{-}(0) & =\left(\sum_{j=m}^{\infty} e^{S_{j}^{-}}\right)^{-1}<\infty \\
\zeta_{\infty, 0}^{-}\left(f_{0, m}^{+}(0)\right) & =\left(\sum_{j=1}^{m-1} e^{-S_{j}^{+}}+\sum_{j=0}^{\infty} e^{S_{j}^{-}}\right)^{-1}<\infty
\end{aligned}
$$

U

$$
\zeta=\left(\sum_{j=1}^{\infty} e^{-S_{j}^{+}}+\sum_{j=0}^{\infty} e^{S_{j}^{-}}\right)^{-1}<\infty \quad \widehat{\mathbf{P}} \text {-п.н. }
$$

Из этих соотношений несложно вывести, что при $n \rightarrow \infty$ для $m \geqslant 0$

и

$$
h_{-m}^{(n)}(s) \stackrel{w(\mathbf{P}, \widehat{\mathbf{P}})}{\longmapsto} \frac{s\left(\zeta_{\infty, m}^{-}(0)-\zeta\right)}{\zeta_{\infty, m}^{-}(0)-s \zeta} \quad \widehat{\mathbf{P}}_{- \text {-ा.н. }}
$$

$$
h_{m}^{(n)}(s) \stackrel{w(\mathbf{P}, \widehat{\mathbf{P}})}{\longmapsto} \frac{s\left(\zeta_{\infty, 0}^{-}\left(f_{0, m}^{+}(0)\right)-\zeta\right)}{\zeta_{\infty, 0}^{-}\left(f_{0, m}^{+}(0)\right)-s \zeta} \quad \widehat{\mathbf{P}} \text {-п.н. }
$$

В частности,

$$
\lim _{n \rightarrow \infty} \mathbf{P}_{\pi}(B(n)-\tau(n)= \pm m) \stackrel{d}{=} \zeta^{-1} e^{\mp S_{m}^{ \pm}}
$$


Рассмотрим теперь конечномерные распределения процесса $\{Z(k, n)$, $0 \leqslant k \leqslant n\}$. Для $0 \leqslant k<l \leqslant n$ положим

$$
V_{k, l}^{(n)}\left(s_{k+1}, \ldots, s_{l}\right):=\mathbf{E}_{\pi}\left[\prod_{i=k+1}^{l} s_{i}^{Z(i, n)} \mid Z(k, n)=1\right] .
$$

Следующая лемма показывает, что процесс $\{Z(k, n), 0 \leqslant k \leqslant n\}$ может рассматриваться как неоднородный процесс Гальтона-Ватсона в случайной среде с производящими функциями $\left\{w_{k, n}(s), 0 \leqslant k \leqslant n\right\}$ (см. определение (21)).

Лемма 1. Имеет место соотношение

$$
\begin{aligned}
& V_{k, l}^{(n)}\left(s_{k+1}, \ldots, s_{l}\right) \\
& \quad=w_{k, n}\left(s_{k+1} w_{k+1, n}\left(s_{k+2} w_{k+2, n}\left(\ldots\left(s_{l-1} w_{l-1, n}\left(s_{l}\right)\right) \ldots\right)\right)\right) .
\end{aligned}
$$

Д ок а з а т ел ь с т в о. Для $k=n-1$ и $l=n$ утверждение леммы следует из (22). Предполагая теперь, что $0 \leqslant k<n-1$ и $l=n$, и замечая, что для $j \geqslant 1$

$$
\begin{aligned}
\mathbf{E}_{\pi}\left[\prod_{i=k+2}^{n} s_{i}^{Z(i, n)} \mid Z(k, n)=1 ; Z(k+1, n)=j\right] \\
=\mathbf{E}_{\pi}\left[\prod_{i=k+2}^{n} s_{i}^{Z(i, n)} \mid Z(k+1, n)=j\right] \\
=\left(\mathbf{E}_{\pi}\left[\prod_{i=k+2}^{n} s_{i}^{Z(i, n)} \mid Z(k+1, n)=1\right]\right)^{j}=\left(V_{k+1, n}^{(n)}\left(s_{k+2}, \ldots, s_{n}\right)\right)^{j},
\end{aligned}
$$

получаем

$$
\begin{aligned}
& V_{k, n}^{(n)}\left(s_{k+1}, s_{k+2}, \ldots, s_{n}\right) \\
& \quad=\mathbf{E}_{\pi}\left[s_{k+1}^{Z(k+1, n)} \mathbf{E}_{\pi}\left[\prod_{i=k+2}^{n} s_{i}^{Z(i, n)} \mid Z(k+1, n) ; Z(k, n)=1\right] \mid Z(k, n)=1\right] \\
& \quad=\mathbf{E}_{\pi}\left[s_{k+1}^{Z(k+1, n)}\left(V_{k+1, n}^{(n)}\left(s_{k+2}, \ldots, s_{n}\right)\right)^{Z(k+1, n)} \mid Z(k, n)=1\right] \\
& \quad=w_{k, n}\left(s_{k+1} V_{k+1, n}^{(n)}\left(s_{k+2}, \ldots, s_{n}\right)\right) .
\end{aligned}
$$

Отсюда, используя индукционный переход, легко получить утверждение леммы для $0 \leqslant k<l=n$. Полагая $s_{l+1}=\cdots=s_{n}=1$ в только что доказанном утверждении, мы приходим к нужному нам утверждению для случая $0 \leqslant k<l<n$. Лемма доказана.

Для $0 \leqslant k_{0}<k_{1}<\cdots<k_{p} \leqslant n$ положим

$$
W_{k_{0}, k_{1}, \ldots, k_{p}}^{(n)}\left(s_{1}, \ldots, s_{p}\right):=\mathbf{E}_{\pi}\left[\prod_{i=1}^{p} s_{i}^{Z\left(k_{i}, n\right)} \mid Z\left(k_{0}, n\right)=1\right] .
$$


Заметим, что

$$
W_{k_{0}, k_{1}}^{(n)}(s)=1-\frac{1-f_{k_{0}, k_{1}}\left(1-(1-s)\left(1-f_{k_{1}, n}(0)\right)\right)}{1-f_{k_{0}, n}(0)} .
$$

Из леммы 1 вытекает следующее утверждение.

Следствие 2. Для любых $0 \leqslant k_{0}<k_{1}<\cdots<k_{p} \leqslant n$

$$
\begin{aligned}
& W_{k_{0}, k_{1}, \ldots, k_{p}}^{(n)}\left(s_{1}, s_{2}, \ldots, s_{p}\right) \\
& \quad=W_{k_{0}, k_{1}}^{(n)}\left(s_{1} W_{k_{1}, k_{2}}^{(n)}\left(s_{2} W_{k_{2}, k_{3}}^{(n)}\left(\ldots\left(s_{p-1} W_{k_{p-1}, k_{p}}^{(n)}\left(s_{p}\right)\right) \ldots\right)\right)\right) .
\end{aligned}
$$

Для $m \in \mathbf{N}_{0}$ положим

$$
\begin{aligned}
w_{-m}(s) & :=\frac{f_{m}^{-}\left(f_{m-1,0}^{-}\left(q^{+}\right)+s\left(1-f_{m-1,0}^{-}\left(q^{+}\right)\right)\right)-f_{m, 0}^{-}\left(q^{+}\right)}{1-f_{m, 0}^{-}\left(q^{+}\right)} \\
w_{m}(s) & :=\frac{f_{m}^{+}\left(q_{m+1}^{+}+s\left(1-q_{m+1}^{+}\right)\right)-q_{m}^{+}}{1-q_{m}^{+}}
\end{aligned}
$$

и для $k<l$, где $k, l \in \mathbf{Z}$, обозначим

$$
h_{k, l}\left(s_{k}, s_{k+1}, \ldots, s_{l}\right):=h_{k}\left(s_{k} w_{k+1}\left(s_{k+1} w_{k+2}\left(\ldots\left(s_{l-1} w_{l}\left(s_{l}\right)\right) \ldots\right)\right)\right) \text {. }
$$

Теперь мы можем сформулировать многомерный вариант теоремы 1.

Теорема 3. Если выполнень условия $A 1-A 2$, то для любого $m \in \mathbf{N}_{0}$

$$
V_{\tau(n)-m, \tau(n)+m}^{(n)}\left(s_{-m}, s_{-(m-1)}, \ldots, s_{m}\right) \stackrel{w(\mathbf{P}, \widehat{\mathbf{P}})}{\longmapsto} h_{-m, m}\left(s_{-m}, s_{-(m-1)}, \ldots, s_{m}\right) .
$$

Д ок а з а т е л с с в о. Утверждение теоремы нетрудно вывести из леммы 1 и теорем 1 и 2 , и мы опускаем подробное доказательство.

Мы завершим этот раздел следуюшим нестрогим описанием наших результатов. В силу следствия 1

$$
\Delta_{m}:=h_{m}^{\prime}(0)-h_{m+1}^{\prime}(0) \stackrel{d}{=} \lim _{n \rightarrow \infty} \mathbf{P}_{\pi}(B(n)=\tau(n)+m),
$$

где, напомним, $B(n)$ - момент рождения ближайшего общего предка всех частиц, существующих в популяции в момент времени $n$. Теорема 3 , следствие 1 и определение (26) показывают, что при $n \rightarrow \infty$ конечномерные распределения критического редуцированного процесса $\{Z(\tau(n)+$ $m, n), m \in \mathbf{Z}\}$ в случайной среде сходятся (в смысле $w(\mathbf{P}, \widehat{\mathbf{P}})$-сходимости) к соответствующим конечномерным распределениям следующего нестандартного ветвящегося процесса $\{\Xi(m), m \in \mathbf{Z}\}$ в случайной среде, заданного на вероятностном пространстве $\left(\Omega^{-} \times \Omega^{+}, \mathscr{F}^{-} \times \mathscr{F}^{+}, \widehat{\mathbf{P}}\right)$. Для каждого $m \in \mathbf{Z}$ производящая функция закона размножения частиц $m$-го поколения равняется $w_{m}(s)$. Процесс начинается в $-\infty$ с одной частицы, которая с вероятностью $\Delta_{m_{0}}$ умирает в момент времени $m_{0} \in(-\infty,+\infty)$, производя потомство в соответствии с производящей 
функцией $w_{m_{0}}(s)$. Начиная с этого момента процесс развивается как ветвящийся процесс в случайной среде, в котором частицы, существующие в момент времени $m>m_{0}$, размножаются по закону, задаваемому производящей функцией $w_{m}(s)$. Это описание еще раз подчеркивает существенное отличие наших результатов от соответствующих предельных результатов для обычных редуцированных докритических и критических процессов Гальтона-Ватсона, установленных в [19] и [15].

3. Свойства редуцированных процессов в моменты времени, расположенные существенно правее $\tau(n)$. В этом разделе мы изучим поведение величины $Z(\tau(n)+m, n)$ в ситуации, когда $m=m(n) \rightarrow \infty$ при $n \rightarrow \infty$. Для $0 \leqslant k \leqslant n$ положим

$$
\beta_{n}(k):=\frac{1}{\mathbf{E}_{\pi}[Z(k, n) \mid Z(n)>0]}=\frac{e^{-S_{k}}\left(1-f_{0, n}(0)\right)}{1-f_{k, n}(0)} .
$$

В частности,

$$
\mathbf{E}_{\pi}[Z(n) \mid Z(n)>0]=\frac{e^{S_{n}}}{1-f_{0, n}(0)}=\frac{1}{\beta_{n}(n)} .
$$

Обозначим

$$
\begin{aligned}
\Phi^{(n)}(\lambda) & :=\mathbf{E}_{\pi}\left[e^{-\lambda Z(n) \beta_{n}(n)} \mid Z(n)>0\right] \\
& =\int_{0}^{\infty} e^{-\lambda x} \mathbf{P}_{\pi}\left(Z(n) \beta_{n}(n) \in d x \mid Z(n)>0\right)=: \int_{0}^{\infty} e^{-\lambda x} \mathscr{M}^{(n)}(d x) .
\end{aligned}
$$

Для меры $\mathscr{M} \in \mathbf{M}$ и функции $J: \mathbf{R} \rightarrow \mathbf{R}$ положим $\mathscr{M}[J]:=\int J(x) \mathscr{M}(d x)$, если указанный интеграл существует.

Следующее утверждение является комбинацией следствия 1 и теоремы 1 , доказанных в работе [10].

Теорема 4. Если выполнень условия $A 1-A 2$, то существует случайная мера $\mathscr{M}$ (являюшаяся с вероятностью 1 собственной и невигожденной) такая, что

$$
\mathscr{M}^{(n)} \stackrel{w(\mathbf{P}, \widehat{\mathbf{P}})}{\longmapsto} \mathscr{M}, \quad n \rightarrow \infty
$$

или же, в терминах преобразований Лапласа,

$$
\Phi^{(n)}(\lambda) \stackrel{w(\mathbf{P}, \widehat{\mathbf{P}})}{\longmapsto} \Phi(\lambda):=\int_{0}^{\infty} e^{-\lambda x} \mathscr{M}(d x), \quad n \rightarrow \infty,
$$

где (28) (так же, как и (29)) означает, что

$$
\mathbf{E}\left[g\left(\mathscr{M}^{(n)}[J]\right)\right] \longrightarrow \widehat{\mathbf{E}}[g(\mathscr{M}[J])], \quad n \rightarrow \infty,
$$

для любьх неслучайных непрерывных функиий $J, g: \mathbf{R} \rightarrow \mathbf{R}$ с компактньми носителями. 
Для $m \in \mathbf{Z}$ обозначим

$$
\mathscr{M}_{m}^{(n)}(d x):=\mathbf{P}_{\pi}\left(Z(\tau(n)+m, n) \beta_{n}(\tau(n)+m) \in d x \mid Z(n)>0\right)
$$

и

$$
Y_{m}^{(n)}(\lambda):=\mathbf{E}_{\pi}\left[e^{-\lambda Z(\tau(n)+m, n) \beta_{n}(\tau(n)+m)} \mid Z(n)>0\right]=\int_{0}^{\infty} e^{-\lambda x} \mathscr{M}_{m}^{(n)}(d x),
$$

где по определению $Z(\tau(n)+m, n)=Z(n, n)=Z(n)$, если $\tau(n)+m>n$, и $Z(\tau(n)+m, n)=Z(0)$, если $\tau(n)+m<0$. Очевидно, что

$$
Y_{m}^{(n)}(\lambda)=W_{0, \tau(n)+m}^{(n)}\left(e^{-\lambda \beta_{n}(\tau(n)+m)}\right) .
$$

Теорема 5. Пусть выполнены условия $A 1-A 2$. Если $n \geqslant m=$ $m(n) \rightarrow \infty$ npu $n \rightarrow \infty$, mo

$$
\mathscr{M}_{m}^{(n)} \stackrel{w(\mathbf{P}, \widehat{\mathbf{P}})}{\longmapsto} \mathscr{M}, \quad n \rightarrow \infty,
$$

или, в терминах преобразований Лапласа,

$$
Y_{m}^{(n)}(\lambda) \stackrel{w(\mathbf{P}, \widehat{\mathbf{P}})}{\longmapsto} \Phi(\lambda), \quad n \rightarrow \infty,
$$

где $\mathscr{M}$ и $\Phi(\lambda)$ те же, ито и в теореме 4.

Для доказательства этой теоремы нам потребуется ряд вспомогательных утверждений. Для $l, m, k \in \mathbf{N}$, где $m \leqslant k$, введем следующее обозначение (вспомните соотношения (3)-(4)):

$$
\Gamma(l, m, k):=\frac{e^{-S_{m}^{+}-S_{l}^{-}}\left(1-f_{l, 0}^{-}\left(f_{0, k}^{+}(0)\right)\right)}{1-f_{m, k}^{+}(0)}=\frac{e^{-S_{m}^{+}} \zeta_{l}^{-}\left(f_{0, k}^{+}(0)\right)}{1-f_{m, k}^{+}(0)} .
$$

Лемма 2. Если выполнены условия $A 1-A 2$, то $\widehat{\mathbf{P}}-$ п.н.

$$
\lim _{m \rightarrow \infty} \Gamma(l, m, k)=0 .
$$

Д о к а зат е л ь с т о. Ясно, что

$$
\Gamma(l, m, k) \leqslant e^{-S_{m}^{+}}\left(1-f_{m, k}^{+}(0)\right)^{-1} .
$$

В силу леммы 5 работы [10] (см. также [12])

$$
\frac{1}{1-f_{m, k}^{+}(0)} \leqslant e^{-S_{k}^{+}+S_{m}^{+}}+\sum_{j=m}^{k-1} \eta_{j+1}^{+} e^{-S_{j}^{+}+S_{m}^{+}},
$$

где $\eta_{j+1}^{+}=\left(f_{j}^{+}\right)^{\prime \prime}(1) /\left(\left(f_{j}^{+}\right)^{\prime}(1)\right)^{2}$. Поэтому

$$
\Gamma(l, m, k) \leqslant \sum_{j=m}^{\infty}\left(\eta_{j+1}^{+}+1\right) e^{-S_{j}^{+}} .
$$

Согласно лемме 2.7 статьи [4],

$$
\sum_{j=0}^{\infty}\left(\eta_{j+1}^{+}+1\right) e^{-S_{j}^{+}}<\infty \quad \widehat{\mathbf{P}} \text {-п.н. }
$$

Следовательно, $\lim _{m \rightarrow \infty} \Gamma(l, m, k)=0 \widehat{\mathbf{P}}$-п.н. Лемма доказана. 
Для $l, m, k \in \mathbf{N}$, где $m \leqslant k$, обозначим

$$
R_{m}^{(l, k)}(\lambda):=1-\frac{1-f_{l, 0}^{-}\left(f_{0, m}^{+}\left(1-\left(1-e^{-\lambda \Gamma(l, m, k)}\right)\left(1-f_{m, k}^{+}(0)\right)\right)\right)}{1-f_{l, 0}^{-}\left(f_{0, k}^{+}(0)\right)} .
$$

Лемма 3. Если выполнень условия $A 1-A 2$, то $\widehat{\mathbf{P}}_{-}$-н.н.

$$
\lim _{\min (l, m) \rightarrow \infty} R_{m}^{(l, k)}(\lambda)=\Phi(\lambda) .
$$

Д о к а з а т е л ь с т в о. Так как $m \leqslant k$, то соотношение (6) влечет

$$
\lim _{\min (l, m) \rightarrow \infty} \frac{\zeta_{l}^{-}\left(f_{0, m}^{+}(0)\right)}{\zeta_{l}^{-}\left(f_{0, k}^{+}(0)\right)}=1 \quad \widehat{\mathbf{P}} \text {-п.н. }
$$

Из этого предельного представления и предыдущей леммы вытекает, что

$$
\begin{aligned}
\left(1-e^{-\lambda \Gamma(l, m, k)}\right)\left(1-f_{m, k}^{+}(0)\right) & =\lambda \Gamma(l, m, k)\left(1+\varepsilon_{1}(\lambda ; l, m, k)\right)\left(1-f_{m, k}^{+}(0)\right) \\
& =\lambda e^{-S_{m}^{+}} \zeta_{l}^{-}\left(f_{0, k}^{+}(0)\right)\left(1+\varepsilon_{1}(\lambda ; l, m, k)\right) \\
& =\lambda e^{-S_{m}^{+}} \zeta_{l}^{-}\left(f_{0, m}^{+}(0)\right)\left(1+\varepsilon_{2}(\lambda ; l, m, k)\right),
\end{aligned}
$$

где $\widehat{\mathbf{P}}$-п.н. $\lim _{\min (l, m) \rightarrow \infty} \varepsilon_{j}(\lambda ; l, m, k)=0, j=1,2$, для любого $\lambda>0$. Следовательно,

$1-\left(1-e^{-\lambda \Gamma(l, m, k)}\right)\left(1-f_{m, k}^{+}(0)\right)=\exp \left\{-\lambda e^{-S_{m}^{+}} \zeta_{l}^{-}\left(f_{0, m}^{+}(0)\right)\left(1+\varepsilon_{3}(\lambda ; l, m, k)\right)\right\}$, где $\widehat{\mathbf{P}}$-п.н. $\lim _{\min (l, m) \rightarrow \infty} \varepsilon_{3}(\lambda ; l, m, k)=0$ для любого $\lambda>0$. Таким образом,

$$
\begin{aligned}
& R_{m}^{(l, k)}(\lambda) \\
& \quad=1-\frac{1-f_{l, 0}^{-}\left(f_{0, m}^{+}\left(\exp \left\{-\lambda e^{-S_{m}^{+}} \zeta_{l}^{-}\left(f_{0, m}^{+}(0)\right)\left(1+\varepsilon_{3}(\lambda ; l, m, k)\right)\right\}\right)\right)}{\left(1-f_{l, 0}^{-}\left(f_{0, m}^{+}(0)\right)\right)\left(1+\varepsilon_{4}(l, m, k)\right)},
\end{aligned}
$$

где $\widehat{\mathbf{P}}$-п.н. $\lim _{\min (l, m) \rightarrow \infty} \varepsilon_{4}(l, m, k)=0$. Из соотношений $(3.1)$ и $(3.6)$ работы [10] (в слегка измененных обозначениях) следует, что $\widehat{\mathbf{P}}$-п.н. для Bcex $\lambda>0$

$$
\lim _{\min (l, m) \rightarrow \infty} \frac{1-f_{l, 0}^{-}\left(f_{0, m}^{+}\left(\exp \left\{-\lambda e^{-S_{m}^{+}} \zeta_{l}^{-}\left(f_{0, m}^{+}(0)\right)\right\}\right)\right)}{1-f_{l, 0}^{-}\left(f_{0, m}^{+}(0)\right)}=1-\Phi(\lambda) .
$$

Это равенство и представление (34) влекут утверждение леммы.

Для $0 \leqslant m_{1}<m_{2} \leqslant k$ и $l \in \mathbf{N}_{0}$ положим

$$
Q_{m_{1}, m_{2}}^{(l, k)}(\lambda):=1-\frac{1-f_{m_{1}, m_{2}}^{+}\left(1-\left(1-e^{-\lambda \Gamma\left(i, m_{2}, k\right)}\right)\left(1-f_{m_{2}, k}^{+}(0)\right)\right)}{1-f_{m_{1}, k}^{+}(0)}
$$

Лемма 4. Если выполнень условия $A 1-A 2$, то для любого $\lambda>0$

$$
\lim _{m_{1} \rightarrow \infty} \frac{\ln Q_{m_{1}, m_{2}}^{(l, k)}(\lambda)}{\Gamma\left(l, m_{1}, k\right)}=\lambda \quad \widehat{\mathbf{P}}_{-n . \mu .}
$$


Д о к а з а т е л ь с т в о. Заметим, что

$$
\begin{aligned}
& \frac{1-f_{m_{1}, m_{2}}^{+}\left(1-\left(1-e^{-\lambda \Gamma\left(l, m_{2}, k\right)}\right)\left(1-f_{m_{2}, k}^{+}(0)\right)\right)}{1-f_{m_{1}, k}^{+}(0)} \\
& \leqslant \frac{e^{S_{m_{2}}^{+}-S_{m_{1}}^{+}}\left(1-e^{-\lambda \Gamma\left(l, m_{2}, k\right)}\right)\left(1-f_{m_{2}, k}^{+}(0)\right)}{1-f_{m_{1}, k}^{+}(0)} \\
& \leqslant \frac{\lambda e^{S_{m_{2}}^{+}-S_{m_{1}}^{+} \Gamma\left(l, m_{2}, k\right)\left(1-f_{m_{2}, k}^{+}(0)\right)}}{1-f_{m_{1}, k}^{+}(0)}=\lambda \Gamma\left(l, m_{1}, k\right),
\end{aligned}
$$

и, следовательно, дробь в (35) стремится к нулю $\widehat{\mathbf{P}}$-п.н. при $m_{1} \rightarrow \infty$ согласно лемме 2. Применяя лемму 2 еще раз, мы видим, что

$$
\left(1-e^{-\lambda \Gamma\left(l, m_{2}, k\right)}\right)\left(1-f_{m_{2}, k}^{+}(0)\right)=\lambda e^{-S_{m_{2}}^{+}} \zeta_{l}^{-}\left(f_{0, k}^{+}(0)\right)\left(1+\varepsilon\left(\lambda, l, m_{2}, k\right)\right),
$$

где $\lim _{m_{2} \rightarrow \infty} \varepsilon\left(\lambda, l, m_{2}, k\right)=0 \widehat{\mathbf{P}}$-п.н. Ввиду $(32) S_{m_{2}}^{+} \rightarrow+\infty \widehat{\mathbf{P}}^{+}$-п.н. при $m_{2} \rightarrow \infty$. Следовательно,

$$
e^{-S_{m_{2}}^{+}} \zeta_{l}^{-}\left(f_{0, k}^{+}(0)\right) \leqslant e^{-S_{m_{2}}^{+}} \underset{m_{2} \rightarrow \infty}{\longrightarrow} 0 \quad \widehat{\mathbf{P}} \text {-п.н. }
$$

Полученные результаты, монотонность функции $f_{m_{1}, m_{2}}^{+}(s)$ по $s$ и эквивалентность $\ln (1-x) \sim x$ при $x \rightarrow+0$ убеждают нас в том, что для доказательства леммы достаточно показать, что $\widehat{\mathbf{P}}$-п.н.

$$
\begin{gathered}
\lim _{m_{1} \rightarrow \infty} \frac{1}{\Gamma\left(l, m_{1}, k\right)} \frac{1-f_{m_{1}, m_{2}}^{+}\left(1-\lambda e^{-S_{m_{2}}^{+}} \zeta_{l}^{-}\left(f_{0, k}^{+}(0)\right)\right)}{1-f_{m_{1}, k}^{+}(0)} \\
=\lim _{m_{1} \rightarrow \infty} \frac{1-f_{m_{1}, m_{2}}^{+}\left(1-\lambda e^{-S_{m_{2}}^{+}} \zeta_{l}^{-}\left(f_{0, k}^{+}(0)\right)\right)}{e^{-S_{m_{1}}^{+}} \zeta_{l}^{-}\left(f_{0, k}^{+}(0)\right)}=\lambda .
\end{gathered}
$$

Согласно лемме 5 работы [10] (см. также [12]),

$$
\begin{aligned}
1-f_{m_{1}, m_{2}}^{+}(1-x) & =\left(e^{S_{m_{1}}^{+}-S_{m_{2}}^{+}} x^{-1}+D_{m_{1}, m_{2}}(x)\right)^{-1} \\
& =e^{S_{m_{2}}^{+}-S_{m_{1}}^{+}} x\left(1+x e^{S_{m_{2}}^{+}-S_{m_{1}}^{+}} D_{m_{1}, m_{2}}(x)\right)^{-1},
\end{aligned}
$$

где функция $D_{m_{1}, m_{2}}(x)$ удовлетворяет неравенствам

$$
0 \leqslant D_{m_{1}, m_{2}}(x) \leqslant \sum_{j=m_{1}}^{m_{2}-1} \eta_{j+1}^{+} e^{-\left(S_{j}^{+}-S_{m_{1}}^{+}\right)}:=D .
$$

При $x=\lambda e^{-S_{m_{2}}^{+}} \zeta_{l}^{-}\left(f_{0, k}^{+}(0)\right) \leqslant \lambda e^{-S_{m_{2}}^{+}}$указанные соотношения приводят к оценкам

$$
1 \geqslant \frac{1-f_{m_{1}, m_{2}}^{+}\left(1-\lambda e^{\left.-S_{m_{2}}^{+} \zeta_{l}^{-}\left(f_{0, k}^{+}(0)\right)\right)}\right.}{\lambda e^{-S_{m_{1}}^{+}} \zeta_{l}^{-}\left(f_{0, k}^{+}(0)\right)} \geqslant\left(1+\lambda e^{-S_{m_{1}}^{+}} D\right)^{-1},
$$


где в соответствии с леммой 2.7 работы [4]

$$
e^{-S_{m_{1}}^{+}} D=e^{-S_{m_{1}}^{+}} \sum_{j=m_{1}}^{m_{2}-1} \eta_{j+1}^{+} e^{-\left(S_{j}^{+}-S_{m_{1}}^{+}\right)} \leqslant \sum_{j=m_{1}}^{\infty} \eta_{j+1}^{+} e^{-S_{j}^{+}} \longrightarrow 0 \quad \widehat{\mathbf{P}}-\Pi . \mathrm{H}
$$

при $m_{1} \rightarrow \infty$. Теперь равенство (36) следует из соотношений $(37)-(39)$. Лемма доказана.

Для $p \geqslant 2$ и $0 \leqslant m_{1}<m_{2}<\cdots<m_{p+1} \leqslant k$ положим

$$
Q_{m_{1}, m_{2}, m_{3}}^{(l, k)}\left(\lambda_{2}, \lambda_{3}\right):=Q_{m_{1}, m_{2}}^{(l, k)}\left(\lambda_{2}+\frac{\ln Q_{m_{2}, m_{3}}^{(l, k)}\left(\lambda_{3}\right)}{\Gamma\left(l, m_{2}, k\right)}\right)
$$

и определим рекурсивно

$$
\begin{aligned}
& Q_{m_{1}, m_{2}, \ldots, m_{p+1}}^{(l, k)}\left(\lambda_{2}, \ldots, \lambda_{p+1}\right) \\
& \quad:=Q_{m_{1}, m_{2}, \ldots, m_{p}}^{(l, k)}\left(\lambda_{2}, \ldots, \lambda_{p-1}, \lambda_{p}+\frac{\ln Q_{m_{p}, m_{p+1}}^{(l, k)}\left(\lambda_{p+1}\right)}{\Gamma\left(l, m_{p}, k\right)}\right) .
\end{aligned}
$$

Лемма 5. Если выполнены условия $A 1-A 2$, то $\widehat{\mathbf{P}}-$ п.н. для любых $p \geqslant 2 u \lambda_{2}, \ldots, \lambda_{p}>0$

$$
\lim _{m_{1} \rightarrow \infty} \frac{\ln Q_{m_{1}, m_{2}, \ldots, m_{p}}^{(l, k)}\left(\lambda_{2}, \ldots, \lambda_{p}\right)}{\Gamma\left(l, m_{1}, k\right)}=\sum_{j=2}^{p} \lambda_{j}
$$

Д о к а з а т л ь с т о. Утверждение данной леммы следует из леммы 4 по индукции.

Лемма 6. Если выполнены условия $A 1-A 2$, то $\widehat{\mathbf{P}}-$ п.н. для любых $p \geqslant 2, \lambda_{1}, \lambda_{2}, \ldots, \lambda_{p}>0 u 0 \leqslant m_{1}<m_{2}<\cdots<m_{p} \leqslant k$

$$
\lim _{\min \left(l, m_{1}\right) \rightarrow \infty} R_{m_{1}}^{(l, k)}\left(\lambda_{1}+\frac{\ln Q_{m_{1}, m_{2}, \ldots, m_{p}}^{(l, k)}\left(\lambda_{2}, \ldots, \lambda_{p}\right)}{\Gamma\left(l, m_{1}, k\right)}\right)=\Phi\left(\sum_{j=1}^{p} \lambda_{j}\right) .
$$

Д о к а з а т л ь с т в о. Утверждение леммы следует из лемм 5 , 4 и 3.

Доказательст в о те о ремы 5. Из (25) и (31) вытекает, что

$$
\begin{aligned}
Y_{m}^{(n)}(\lambda) & =\int_{0}^{\infty} e^{-\lambda x} \mathscr{M}_{m}^{(n)}(d x) \\
& =1-\frac{1-f_{0, \tau(n)+m}\left(1-\left(1-e^{-\lambda \beta_{n}(\tau(n)+m)}\right)\left(1-f_{\tau(n)+m, n}(0)\right)\right)}{1-f_{0, n}(0)}
\end{aligned}
$$

Покажем, что для любых неслучайных непрерывных функций $J, g$ : $\mathbf{R} \rightarrow \mathbf{R}$ с компактными носителями

$$
\lim _{m \rightarrow \infty} \mathbf{E}\left[g\left(\mathscr{M}_{m}^{(n)}[J]\right)\right]=\widehat{\mathbf{E}}[g(\mathscr{M}[J])]
$$

Несложно проверить, используя (40) и (33), что

$$
\mathbf{E}\left[g\left(\mathscr{M}_{m}^{(n)}[J]\right) \mid \tau(n)=l\right]=\mathbf{E}\left[g\left(\mathscr{M}_{m}^{(l, n-l)}[J]\right) \mid \mathscr{A}_{l, n-l}\right],
$$


где $\mathscr{M}_{m}^{(l, n-l)}$ - мера с преобразованием Лапласа $R_{m}^{(l, n-l)}(\lambda)$. Согласно лемме $3, \widehat{\mathbf{P}}$-п.н. $\mathscr{M}_{m}^{(l, n-l)}[J] \rightarrow \mathscr{M}[J]$ при $\min (l, m) \rightarrow \infty$. Отсюда и из леммы 3 работы [10] вытекает, что для любых неслучайных непрерывных функций $J, g: \mathbf{R} \rightarrow \mathbf{R}$ с компактными носителями

$$
\begin{aligned}
& \lim _{\min (l, m) \rightarrow \infty} \mathbf{E}\left[g\left(\mathscr{M}_{m}^{(n)}[J]\right) \mid \tau(n)=l\right] \\
& \quad=\lim _{\min (l, m) \rightarrow \infty} \mathbf{E}\left[g\left(\mathscr{M}_{m}^{(l, n-l)}[J]\right) \mid \mathscr{A}_{l, n-l}\right]=\widehat{\mathbf{E}}[g(\mathscr{M}[J])] .
\end{aligned}
$$

Для завершения доказательства (41) осталось лишь применить при $\varepsilon \in\left(0, \frac{1}{2}\right)$ формулу полной вероятности

$$
\begin{aligned}
\mathbf{E}\left[g\left(\mathscr{M}_{m}^{(n)}[J]\right)\right]= & \sum_{0 \leqslant l \leqslant n \varepsilon} \mathbf{E}\left[g\left(\mathscr{M}_{m}^{(l, n-l)}[J]\right) \mid \mathscr{A}_{l, n-l}\right] \mathbf{P}(\tau(n)=l) \\
& +\sum_{n \varepsilon<l<n(1-\varepsilon)} \mathbf{E}\left[g\left(\mathscr{M}_{m}^{(l, n-l)}[J]\right) \mid \mathscr{A}_{l, n-l}\right] \mathbf{P}(\tau(n)=l) \\
& +\sum_{n(1-\varepsilon) \leqslant l \leqslant n} \mathbf{E}\left[g\left(\mathscr{M}_{m}^{(l, n-l)}[J]\right) \mid \mathscr{A}_{l, n-l}\right] \mathbf{P}(\tau(n)=l),
\end{aligned}
$$

устремить $n \rightarrow \infty$, применить для оценки средней суммы равенство (42), а для оценки первой и последней сумм воспользоваться ограниченностью функции $g$ и заметить, что согласно обобщенному закону арксинуса

$$
\lim _{\varepsilon \downarrow \rightarrow 0} \lim _{n \rightarrow \infty} \mathbf{P}(\tau(n) \in[0, n \varepsilon] \bigcup[n(1-\varepsilon), n])=0 .
$$

Теорема 5 доказана.

Теперь мы получим многомерное обобщение теоремы 5. Пусть для $0 \leqslant m_{1}<m_{2}<\cdots<m_{p} \leqslant n-\tau(n)$

$$
Y_{m_{1}, \ldots, m_{p}}^{(n)}\left(\lambda_{1}, \ldots, \lambda_{p}\right):=\mathbf{E}_{\pi}\left[\prod_{i=1}^{p} e^{-\lambda_{i} Z\left(\tau(n)+m_{i}, n\right) \beta_{n}\left(\tau(n)+m_{i}\right)} \mid Z(0, n)=1\right] .
$$

Полагая $s_{i}=s_{i}\left(\lambda_{i}, n\right):=\exp \left\{-\lambda_{i} \beta_{n}\left(\tau(n)+m_{i}\right)\right\}, i=1, \ldots, p$, несложно увидеть, что

$$
Y_{m_{1}, \ldots, m_{p}}^{(n)}\left(\lambda_{1}, \ldots, \lambda_{p}\right)=W_{0, \tau(n)+m_{1}, \ldots, \tau(n)+m_{p}}^{(n)}\left(s_{1}, \ldots, s_{p}\right)
$$

и, следовательно,

$$
Y_{m_{1}, \ldots, m_{p}}^{(n)}\left(\lambda_{1}, \ldots, \lambda_{p}\right)=W_{0, \tau(n)+m_{1}}^{(n)}\left(s_{1} W_{\tau(n)+m_{1}, \ldots, \tau(n)+m_{p}}^{(n)}\left(s_{2}, \ldots, s_{p}\right)\right) .
$$

Теорема 6. Пусть выполнены условия $A 1-A 2$. Если $m_{1}=$ $m_{1}(n) \rightarrow \infty$ npu $n \rightarrow \infty$, mo

$$
Y_{m_{1}, \ldots, m_{p}}^{(n)}\left(\lambda_{1}, \ldots, \lambda_{p}\right) \stackrel{w(\mathbf{P}, \widehat{\mathbf{P}})}{\longmapsto} \Phi\left(\sum_{i=1}^{p} \lambda_{i}\right) .
$$


Д о к а з а т е л ь с т в о. Случай $p=1$ был рассмотрен в теореме 5 . Предположим теперь, что $p \geqslant 2$. Обозначим $\mathscr{M}_{m_{1}, \ldots, m_{p}}^{(n)}\left(d x_{1}, \ldots, d x_{p}\right)$ меру с преобразованием Лапласа $Y_{m_{1} \ldots, m_{p}}^{(n)}\left(\lambda_{1}, \ldots, \lambda_{p}\right)$. Покажем, что для любых неслучайных непрерывных функций $J: \mathbf{R}^{p} \rightarrow \mathbf{R}^{p}$ и $g: \mathbf{R} \rightarrow \mathbf{R}$ с компактными носителями

$$
\begin{aligned}
& \lim _{m_{1}(n) \rightarrow \infty} \mathbf{E}\left[g\left(\int J\left(x_{1}, \ldots, x_{p}\right) \mathscr{M}_{m_{1}, \ldots, m_{p}}^{(n)}\left(d x_{1}, \ldots, d x_{p}\right)\right)\right] \\
& =\widehat{\mathbf{E}}\left[g\left(\int J\left(x_{1}, \ldots, x_{p}\right) \mathscr{M}\left(d x_{1}, \ldots, d x_{p}\right)\right)\right]
\end{aligned}
$$

где $\mathscr{M}\left(d x_{1}, \ldots, d x_{p}\right)$ - мера с преобразованием Лапласа $\Phi\left(\sum_{i=1}^{p} \lambda_{i}\right)$.

Используя $(43),(25),(44)$ и следствие 2 , можно показать, что

$$
\begin{aligned}
\mathbf{E}[g & \left.\left(\int J\left(x_{1}, \ldots, x_{p}\right) \mathscr{M}_{m_{1}, \ldots, m_{p}}^{(n)}\left(d x_{1}, \ldots, d x_{p}\right)\right) \mid \tau(n)=l\right] \\
& =\mathbf{E}\left[g\left(\int J\left(x_{1}, \ldots, x_{p}\right) \mathscr{M}_{m_{1}, \ldots, m_{p}}^{(l, n-l)}\left(d x_{1}, \ldots, d x_{p}\right)\right) \mid \mathscr{A}_{l, n-l}\right],
\end{aligned}
$$

где $\mathscr{M}_{m_{1}, \ldots, m_{p}}^{(l, n-l)}\left(d x_{1}, \ldots, d x_{p}\right)$ - мера с преобразованием Лапласа

$$
R_{m_{1}}^{(l, n-l)}\left(\lambda_{1}+\frac{\ln Q_{m_{1}, m_{2}, \ldots, m_{p}}^{(l, k)}\left(\lambda_{2}, \ldots, \lambda_{p}\right)}{\Gamma\left(l, m_{1}, k\right)}\right) .
$$

Из леммы 6 следует, что $\widehat{\mathbf{P}}$-п.н. при $\min \left(l, m_{1}\right) \rightarrow \infty$

$$
\begin{gathered}
\int J\left(x_{1}, \ldots, x_{p}\right) \mathscr{M}_{m_{1}, \ldots, m_{p}}^{(l, n-l)}\left(d x_{1}, \ldots, d x_{p}\right) \\
\stackrel{w}{\longrightarrow} \int J\left(x_{1}, \ldots, x_{p}\right) \mathscr{M}\left(d x_{1}, \ldots, d x_{p}\right) .
\end{gathered}
$$

Отсюда и из леммы 3 в [10] вытекает, что при выборе $J$ и $g$ в соответствии в нашими требованиями

$$
\begin{aligned}
& \lim _{\min (l, m) \rightarrow \infty} \mathbf{E}\left[g\left(\int J\left(x_{1}, \ldots, x_{p}\right) \mathscr{M}_{m_{1}, \ldots, m_{p}}^{(l, n-l)}\left(d x_{1}, \ldots, d x_{p}\right)\right) \mid \mathscr{A}_{l, n-l}\right] \\
& \quad=\widehat{\mathbf{E}}\left[g\left(\int J\left(x_{1}, \ldots, x_{p}\right) \mathscr{M}\left(d x_{1}, \ldots, d x_{p}\right)\right)\right] .
\end{aligned}
$$

Теперь для завершения доказательства теоремы 6 осталось повторить конец доказательства теоремы 5.

4. Редуцированные процессы в моменты времени $n t$, $0<t<1$. До сих пор мы изучали поведение редуцированного процесса или в окрестности случайной точки $\tau(n)$, или в моменты времени, находящиеся значительно правее этой случайной точки. Используя полученные результаты, мы можем теперь доказать условную предельную теорему, описываюшую свойства редуцированного процесса в неслучайныее (но растущие) моменты времени. Для $t \in(0,1]$ положим

$$
\mathscr{M}^{(n)}(t ; d x):=\mathbf{P}_{\pi}\left(Z(n t, n) \beta_{n}(n t) \in d x \mid Z(n)>0\right) .
$$


Пусть

$$
\Phi^{(n)}(t, \lambda):=\mathbf{E}_{\pi}\left[e^{-\lambda Z(n t, n) \beta_{n}(n t)} \mid Z(n)>0\right]=\int_{0}^{\infty} e^{-\lambda x} \mathscr{M}^{(n)}(t ; d x) .
$$

Обозначим $\delta_{1}$ вероятностную меру с единичным атомом в точке 1 , и пусть $\tau$ - случайная величина, задаваемая соотношением (1).

Теорема 7. Если выполнены условия $A 1$ и $A 2$, то для любого $t \in(0,1)$

$$
\mathscr{M}^{(n)}(t ; \cdot) \stackrel{w(\mathbf{P}, \widehat{\mathbf{P}})}{\longmapsto} \mathscr{M} I\{\tau \leqslant t\}+\delta_{1} I\{\tau>t\}, \quad n \rightarrow \infty,
$$

где случайная мера $\mathscr{M}$ и случайная величина $\tau$ не зависят друг от друга, или, в терминах преобразований Лапласа,

$$
\Phi^{(n)}(t, \lambda) \stackrel{w(\mathbf{P}, \widehat{\mathbf{P}})}{\longmapsto} \Phi(\lambda) I\{\tau \leqslant t\}+e^{-\lambda} I\{\tau>t\}, \quad n \rightarrow \infty .
$$

3 а м е ч а н и е 2. Можно показать, что если мера $\mathbb{P}$ сконцентрирована на множестве геометрических вероятностных производящих функций, то выражение, стоящее в правой части (45), имеет вид $(1+\lambda)^{-1} I\{\tau \leqslant t\}+e^{-\lambda} I\{\tau>t\}$.

Д ок а з а т ел ь с т в о т е о ре м ы 7 . Для $\varepsilon>0$ введем множество

$$
\mathscr{C}_{\varepsilon}:=\{|\tau(n)-n t| \leqslant n t \varepsilon\} \bigcup\{0 \leqslant \tau(n) \leqslant n \varepsilon\} \bigcup\{(1-\varepsilon) \leqslant \tau(n) \leqslant n\} .
$$

Для любого достаточно малого $\varepsilon>0$ имеем

$$
\begin{aligned}
\mathscr{M}^{(n)}(t ; \cdot)= & \mathscr{M}^{(n)}(t ; \cdot) I\{n \varepsilon<\tau(n)<n t(1-\varepsilon)\}+\mathscr{M}^{(n)}(t ; \cdot) I\left\{\mathscr{C}_{\varepsilon}\right\} \\
& +\mathscr{M}^{(n)}(t ; \cdot) I\{n t(1+\varepsilon)<\tau(n)<n(1-\varepsilon)\}
\end{aligned}
$$

что приводит к равенству

$$
\begin{aligned}
\mathbf{E}\left[g\left(\mathscr{M}^{(n)}(t ; \cdot)[J]\right)\right]= & \mathbf{E}\left[g\left(\mathscr{M}_{n t-\tau(n)}^{(n)}[J]\right) I\{n \varepsilon<\tau(n)<n t(1-\varepsilon)\}\right] \\
& +\mathbf{E}\left[g\left(\mathscr{M}^{(n)}(t ; \cdot)[J]\right) I\left\{\mathscr{C}_{\varepsilon}\right\}\right] \\
& +\mathbf{E}\left[g\left(\mathscr{M}_{n t-\tau(n)}^{(n)}[J]\right) I\{n t(1+\varepsilon)<\tau(n)<n(1-\varepsilon)\}\right],
\end{aligned}
$$

где мера $\mathscr{M}_{m}^{(n)}$ та же, что и в (30). Ввиду теоремы 5 и обобщенного закона арксинуса

$$
\begin{aligned}
& \lim _{\varepsilon \downarrow \rightarrow 0} \lim _{n \rightarrow \infty} \mathbf{E}\left[g\left(\mathscr{M}_{n t-\tau(n)}^{(n)}[J]\right) I\{n t \varepsilon<\tau(n)<n t(1-\varepsilon)\}\right] \\
& \quad=\lim _{\varepsilon \downarrow \rightarrow 0} \lim _{n \rightarrow \infty} \mathbf{E}\left[g\left(\mathscr{M}_{n t-\tau(n)}^{(n)}[J]\right) I\{n t \varepsilon<n-\tau(n)<n t(1-\varepsilon)\}\right] \\
& \quad=\lim _{\varepsilon \downarrow \rightarrow 0} \lim _{n \rightarrow \infty} \sum_{m=[n t \varepsilon]+1}^{[n t(1-\varepsilon)]-1} \mathbf{E}\left[g\left(\mathscr{M}_{m}^{(n)}[J]\right) \mid \tau(n)=n t-m\right] \mathbf{P}(\tau(n)=n t-m) \\
& =\mathbf{E}[g(\mathscr{M}[J])] \lim _{\varepsilon \downarrow \rightarrow 0} \mathbf{P}(n t \varepsilon<\tau(n)<n t(1-\varepsilon)) \\
& \quad=\mathbf{E}[g(\mathscr{M}[J])] \mathbf{P}(\tau \leqslant t) .
\end{aligned}
$$


Используя обобщенный закон арксинуса еще раз, получаем

$$
\begin{aligned}
& \lim _{\varepsilon \downarrow \rightarrow 0} \limsup _{n \rightarrow \infty} \mathbf{E}\left[g\left(\mathscr{M}^{(n)}(t ; \cdot)[J]\right) I\left\{\mathscr{C}_{\varepsilon}\right\}\right] \\
& \quad \leqslant \sup _{x} g(x) \lim _{\varepsilon \downarrow \rightarrow 0} \limsup _{n \rightarrow \infty} \mathbf{P}\left(\mathscr{C}_{\varepsilon}\right) \\
& \quad=\sup _{x} g(x) \lim _{\varepsilon \downarrow \rightarrow 0} \mathbf{P}(\tau \in[0, \varepsilon] \bigcup[t(1-\varepsilon), t(1+\varepsilon)] \bigcup(1-\varepsilon, 1])=0 .
\end{aligned}
$$

Наконец, аналогично (46)

$$
\begin{aligned}
& \lim _{\varepsilon \downarrow \rightarrow 0} \lim _{n \rightarrow \infty} \mathbf{E}\left[g\left(\mathscr{M}_{n t-\tau(n)}^{(n)}[J]\right) I\{n t(1+\varepsilon)<\tau(n)<n(1-\varepsilon)\}\right] \\
& \quad=\mathbf{E}[g(J(1))] \mathbf{P}(\tau \leqslant t)
\end{aligned}
$$

ввиду следствия 4 статьи [10] и следствия 1 настоящей работы, согласно которым

$\lim _{\varepsilon \downarrow \rightarrow 0} \limsup _{n \rightarrow \infty} \mathbf{P}\left(\beta_{n}(n t) \notin[1-\varepsilon, 1] ; n t(1+\varepsilon)<\tau(n)<n(1-\varepsilon)\right)=0$.

Комбинация полученных оценок доказывает теорему 7.

Проводя похожие рассуждения, можно доказать следующую многомерную предельную теорему.

Теорема 8. Пусть $0<t_{1}<\cdots<t_{k} \leqslant 1$. Если вылолненьи условия $A 1$ и A2, то для любого набора $\lambda_{i} \geqslant 0, i=1,2, \ldots, k, n p u n \rightarrow \infty$ $\mathbf{E}_{\pi}\left[\exp \left\{-\sum_{i=1}^{k} \lambda_{i} Z\left(n t_{i}, n\right) \beta_{n}\left(n t_{i}\right)\right\} \mid Z_{n}>0\right] \stackrel{w(\mathbf{P}, \widehat{\mathbf{P}})}{\longmapsto} \Phi\left(\sum_{i=i_{\tau}}^{k} \lambda_{i}\right) e^{-\sum_{i^{2}=1}^{i_{\tau}-1} \lambda_{i}}$, zдe $i_{\tau}=\min \left\{i: \tau<t_{i}\right\}$.

З а м е ч а н и е 3 . Применяя рассуждения, аналогичные использовавшимся при доказательстве следствия 1.3 в [6], можно показать, что если вероятностные производящие функции законов размножения являются геометрическими с вероятностью 1 , то

$$
\Phi\left(\sum_{i=i_{\tau}}^{k} \lambda_{i}\right)=\frac{1}{1+\sum_{i=i_{\tau}}^{k} \lambda_{i}} \quad \widehat{\mathbf{P}} \text {-п.н. }
$$

Утверждение теоремы 8 допускает следующую не вполне строгую интерпретацию. Конечномерные распределения последовательности процессов $\left\{Z(n t, n) \beta_{n}(n t), 0 \leqslant t \leqslant 1\right\}$ сходятся при $n \rightarrow \infty$ к конечномерным распределениям процесса $\left\{Z_{\infty}(t), 0 \leqslant t \leqslant 1\right\}$, который с вероятностью 1 имеет лишь один скачок на интервале $[0,1]$. А именно,

$$
Z_{\infty}(t)=I\{\tau \leqslant t\}+Z_{\infty} I\{\tau>t\}
$$

где $\tau$ - случайная точка, распределенная согласно обобщенному закону арксинуса с параметром $\rho$ на интервале $[0,1]$, а (случайное) преобразование Лапласа распределения случайной величины $Z_{\infty}$ равно $\Phi(\lambda)$, причем 
величины $\tau$ и $Z_{\infty}$ независимы. В частности, если вероятностные производящие функции законов размножения частиц являются геометрическими с вероятностью 1 , то $\widehat{\mathbf{P}}$-п.н. случайная величина $Z_{\infty}$ имеет экспоненциальное распределение с параметром 1.

5. Случай устойчивых сопровождающих случайных блужданий. Теорема 7 и определение (27) показывают, что случайная величина $Z(n t, n)$ пропорциональна (с точностью до случайного положительного и конечного множителя) величине $\mathbf{E}_{\pi}[Z(n t, n) \mid Z(n)>0]=$ $1 / \beta_{n}(n t)$. Но что можно сказать об асимптотическом поведении нормирующей функции $\beta_{n}(n t), 0<t<1$, при $n \rightarrow \infty$ ? $\mathrm{K}$ сожалению, нам удалось ответить на этот вопрос лишь в случае, когда условие $A 1$ заменено более сильным ограничением. Именно, будем предполагать, что распределение величин $X_{i}, i=1,2, \ldots$, порождающих сопровождающее случайное блуждание ветвящегося процесса в случайной среде, принадлежит области притяжения устойчивого закона. Для более точного описания наших предположений обозначим $\mathscr{A}=\{\alpha, \theta\}$ подмножество в $\mathbf{R}^{2}$, задаваемое соотношением

$$
\mathscr{A}:=\{0<\alpha<1 ;|\theta|<1\} \bigcup\{1<\alpha<2 ;|\theta| \leqslant 1\} \bigcup\{(1,0) ;(2,0)\} .
$$

Пусть $C(\alpha, \theta)$ - множество функций распределения, принадлежащих области притяжения устойчивого закона с характеристической функцией

$$
\chi(t):=\exp \left\{-c|t|^{\alpha}\left(1+i \theta \frac{t}{|t|} \operatorname{tg} \frac{\pi \alpha}{2}\right)\right\}, \quad c>0, \quad(\alpha, \theta) \in \mathscr{A} .
$$

Условие $A 3$. Существуют $(\alpha, \theta) \in \mathscr{A}$ такие, что $G(x):=\mathbf{P}\{X<$ $x\} \in C(\alpha, \theta)$ и, кроме того, $\mathbf{E} X=0$ при $1<\alpha \leqslant 2$.

Заметим, что при выполнении условия $A 3$

$$
\lim _{n \rightarrow \infty} \mathbf{P}\left\{S_{n}>0\right\}=\frac{1}{2}+\frac{1}{\pi \alpha} \operatorname{arctg}\left(-\theta \operatorname{tg} \frac{\pi \alpha}{2}\right) \in(0,1),
$$

т.е. условие $A 1$ Спицера-Дони также выполнено.

Теорема 9. Если выполнены условия $A 2-A 3$, то для любого $t \in(0,1)$

$$
\left\{e^{S_{\tau(n t, n)}-S_{\tau(n)}} \beta_{n}(n t) \mid \tau(n)<n t\right\} \stackrel{w}{\longrightarrow} \frac{\zeta^{-}\left(q^{+}\right)}{\zeta^{*-}\left(q^{*+}\right)}, \quad n \rightarrow \infty,
$$

где $\left(\zeta^{-}(s), q^{+}\right) \stackrel{d}{=}\left(\zeta^{*-}(s), q^{*}\right)\left(\right.$ см. (2)-(6)) и множество $\left\{\zeta^{-}(s), \zeta^{*-}(s)\right.$, $\left.q^{+}, q^{*}\right\}$ состоит из независимых элементов (случайньх функций и случайных величин).

3 а м е ч а н и е 4. Теоремы 9 и 7 показывают, что если условия $A 2-A 3$ выполнены и момент $\tau(n)$ расположен левее точки наблюдения $n t$, то $Z(n t, n)$ имеет порядок $e^{S_{\tau(n t, n)}-S_{\tau(n)}}$ (с точностью до случайного множителя, отделенного от нуля и бесконечности). 
Появление условия $A 3$ требует введения дополнительных обозначений.

Известно [14], что при выполнении условия $A 3$ существует функция $l(n)$, медленно меняющаяся на бесконечности и такая, что для $w_{n}:=n^{1 / \alpha} l(n), \Gamma:=\min \left\{n \geqslant 1: S_{n} \geqslant 0\right\}$ и $\gamma:=\min \left\{n \geqslant 1: S_{n}<0\right\}$ имеет место следующая условная сходимость при $n \rightarrow \infty$, в пространстве $D[0,1]$, оснащенном топологией Скорохода:

$$
\left\{\widetilde{S}_{n}(t):=\frac{S_{n t}}{w_{n}}, 0 \leqslant t \leqslant 1 \mid \gamma>n\right\} \Longrightarrow\left\{W^{+}(t), 0 \leqslant t \leqslant 1\right\}
$$

и

$$
\left\{\widetilde{S}_{n}(t), 0 \leqslant t \leqslant 1 \mid \Gamma>n\right\} \Longrightarrow\left\{W^{-}(t), 0 \leqslant t \leqslant 1\right\}
$$

где $W^{+}$и $W^{-}$- положительная и отрицательная извилины строго устойчивого процесса Леви порядка $\alpha$.

Лемма 7. Пусть $\left\{T_{n}\right\}_{n \geqslant 1}-$ последовательность равномерно ограниченных случайных величин таких, что для любого $n$ случайная величина $T_{n}$ измерима относительно б-алгебры $\mathscr{F}_{n}$, и пусть $\widetilde{S}_{n}=\widetilde{S}_{n}(1)$. Если выполнено условие $A 3$, то

1) если $\lim _{n \rightarrow \infty} T_{n}=: T$ существует $\widehat{\mathbf{P}}^{+}{ }_{-}$п.н., то

$$
\mathscr{L}\left(\left(T_{n} ; \widetilde{S}_{n}\right) \mid \gamma>n\right) \stackrel{w}{\longrightarrow} \widehat{\mathscr{L}}^{+}(T) \mathscr{L}\left(W^{+}(1)\right), \quad n \rightarrow \infty
$$

2) если $\lim _{n \rightarrow \infty} T_{n}=: T$ cуществует $\widehat{\mathbf{P}}^{-}$-n.н., mo

$$
\mathscr{L}\left(\left(T_{n} ; \widetilde{S}_{n}\right) \mid \Gamma>n\right) \stackrel{w}{\longrightarrow} \widehat{\mathscr{L}}^{-}(T) \mathscr{L}\left(W^{-}(1)\right), \quad n \rightarrow \infty .
$$

Д ок а з а т л в с т в о. Докажем соотношение (47). Согласно лемме 9 работы [10], при выполнении условий леммы 7 для любого $r$

$$
\mathscr{L}\left(\left(T_{r} ; \widetilde{S}_{n}\right) \mid \gamma>n\right) \stackrel{w}{\longrightarrow} \widehat{\mathscr{L}}^{+}\left(T_{r}\right) \mathscr{L}\left(W^{+}(1)\right), \quad n \rightarrow \infty .
$$

Далее, для непрерывных в $\mathbf{R}$ функций $0 \leqslant g_{1}(x) \leqslant c_{1}$ и $0 \leqslant g_{2}(x) \leqslant 1$ имеем

$$
\left|\mathbf{E}\left[g_{1}\left(T_{n}\right) g_{2}\left(\widetilde{S}_{n}\right)-g_{1}\left(T_{r}\right) g_{2}\left(\widetilde{S}_{n}\right) \mid \gamma>n\right]\right| \leqslant \mathbf{E}\left[\left|g_{1}\left(T_{n}\right)-g_{1}\left(T_{r}\right)\right| \mid \gamma>n\right] .
$$

Поскольку $\mathbf{P}^{+}$-п.н. $\lim _{n \rightarrow \infty}\left|g_{1}\left(T_{n}\right)-g_{1}\left(T_{r}\right)\right|=\left|g_{1}(T)-g_{1}\left(T_{r}\right)\right|$, то в силу леммы 2.5 статьи [4] и условий нашей леммы

$$
\lim _{n \rightarrow \infty} \mathbf{E}\left[\left|g_{1}\left(T_{n}\right)-g_{1}\left(T_{r}\right)\right| \mid \gamma>n\right]=\widehat{\mathbf{E}}^{+}\left[\left|g_{1}(T)-g_{1}\left(T_{r}\right)\right|\right]
$$

Отсюда, используя теорему об ограниченной сходимости и непрерывность функции $g_{1}$, получаем

$$
\lim _{r \rightarrow \infty} \widehat{\mathbf{E}}^{+}\left[\left|g_{1}(T)-g_{1}\left(T_{r}\right)\right|\right]=\widehat{\mathbf{E}}^{+}\left[\left|g_{1}(T)-g_{1}(T)\right|\right]=0 .
$$


Объединяя соотношения (49)-(52), находим

$$
\lim _{n \rightarrow \infty} \mathbf{E}\left[g_{1}\left(T_{n}\right) g_{2}\left(\widetilde{S}_{n}\right) \mid \gamma>n\right]=\widehat{\mathbf{E}}^{+}\left[g_{1}(T)\right] \mathbf{E}\left[g_{2}\left(W^{+}(1)\right)\right],
$$

что доказывает первую часть леммы.

Соотношение (48) может быть доказано при помощи аналогичных рассуждений.

Пусть $\mathbf{P}\left(t_{1}, t_{2}\right):=\mathbf{P}\left\{t_{2}^{1 / \alpha} W_{2}^{+}(1)>-t_{1}^{1 / \alpha} W_{1}^{-}(1)\right\}$, где $W_{1}^{-}$и $W_{2}^{+}-$ независимые отрицательная и положительная извилины строго устойчивого процесса Леви порядка $\alpha$.

Лемма 8. Пусть $\left\{T_{r, l}\right\}_{r \geqslant 1, l \geqslant 1}$ - двойная последовательность равномерно ограниченньх случайньх величин таких, что для любых $r, l$ случайная величина $T_{r, l}$ измерима относительно $\sigma$-алгебры $\mathscr{F}_{r}^{-} \times \mathscr{F}_{l}^{+}$. Если выполнено условие $A 3$ u $\lim _{\min (l, r) \rightarrow \infty} T_{r, l}=: T$ существует $\widehat{\mathbf{P}}-n$. н., то для любого $\delta \in\left(0, \frac{1}{2}\right)$

$$
\mathbf{E}\left[T_{n t_{1}, n t_{2}} \mid\left\{S_{n t_{2}}^{+}>-S_{n t_{1}}^{-}\right\} \cap \mathscr{A}_{n t_{1}, n t_{2}}\right] \longrightarrow \widehat{\mathbf{E}}[T], \quad n \rightarrow \infty,
$$

равномерно по $0<\delta \leqslant t_{1}, t_{2} \leqslant 1-\delta$.

Д ок а з а т е л с т в о. Для Фиксированного $l$ имеем

$$
\begin{aligned}
& \left|\mathbf{E}\left[T_{n t_{1}, n t_{2}} I\left\{S_{n t_{2}}^{+}>-S_{n t_{1}}^{-}\right\} \mid \mathscr{A}_{n t_{1}, n t_{2}}\right]-\widehat{\mathbf{E}}[T] \mathbf{P}\left(t_{1}, t_{2}\right)\right| \\
& \leqslant\left|\mathbf{E}\left[\left(T_{n t_{1}, n t_{2}}-T_{l, l}\right) I\left\{S_{n t_{2}}^{+}>-S_{n t_{1}}^{-}\right\} \mid \mathscr{A}_{n t_{1}, n t_{2}}\right]\right| \\
& \quad+\left|\mathbf{E}\left[T_{l, l} I\left\{S_{n t_{2}}^{+}>-S_{n t_{1}}^{-}\right\} \mid \mathscr{A}_{n t_{1}, n t_{2}}\right]-\widehat{\mathbf{E}}\left[T_{l, l}\right] \mathbf{P}\left(t_{1}, t_{2}\right)\right| \\
& \quad+\left|\widehat{\mathbf{E}}\left[T_{l, l}\right]-\widehat{\mathbf{E}}[T]\right| \mathbf{P}\left(t_{1}, t_{2}\right) .
\end{aligned}
$$

Из леммы 10 работы [10] нетрудно вывести, что

$$
\mathbf{E}\left[T_{l, l} I\left\{S_{n t_{2}}^{+}>-S_{n t_{1}}^{-}\right\} \mid \mathscr{A}_{n t_{1}, n t_{2}}\right] \longrightarrow \widehat{\mathbf{E}}\left[T_{l, l}\right] \mathbf{P}\left(t_{1}, t_{2}\right)
$$

при $n \rightarrow \infty$ равномерно по $0<\delta \leqslant t_{1}, t_{2} \leqslant 1-\delta$. В силу условий доказываемой леммы

$$
\lim _{l \rightarrow \infty}\left|\widehat{\mathbf{E}}\left[T_{l, l}\right]-\widehat{\mathbf{E}}[T]\right| \mathbf{P}\left(t_{1}, t_{2}\right) \leqslant \lim _{l \rightarrow \infty}\left|\widehat{\mathbf{E}}\left[T_{l, l}\right]-\widehat{\mathbf{E}}[T]\right| \longrightarrow 0
$$

равномерно по $0<\delta \leqslant t_{1}, t_{2} \leqslant 1-\delta$. Наконец,

$$
\begin{aligned}
& \left|\mathbf{E}\left[\left(T_{l, l}-T_{n t_{1}, n t_{2}}\right) I\left\{S_{n t_{2}}^{+}>-S_{n t_{1}}^{-}\right\} \mid \mathscr{A}_{n t_{1}, n t_{2}}\right]\right| \\
& \quad \leqslant \mathbf{E}\left[\left|T_{l, l}-T_{n t_{1}, n t_{2}}\right| \mid \mathscr{A}_{n t_{1}, n t_{2}}\right]
\end{aligned}
$$

и, следуя доказательству леммы 3 статьи [10], нетрудно понять, что правая часть (56) стремится к нулю равномерно по $0<\delta \leqslant t_{1}, t_{2} \leqslant 1-\delta$, если сначала $n \rightarrow \infty$, а затем $l \rightarrow \infty$. Для завершения доказательства леммы достаточно объединить соотношения (53)-(56) и заметить, что $\mathbf{P}\left(t_{1}, t_{2}\right) \geqslant \mathbf{P}(1-\delta, \delta)>0$ при $0<\delta \leqslant t_{1}, t_{2} \leqslant 1-\delta$. 
Пусть

$$
\begin{array}{ll}
u_{1}(n t):=\frac{1-f_{0, \tau(n t)}(s)}{e^{S_{\tau(n t)}} ;} & u_{2}(n t):=f_{\tau(n t), n t}(0) \\
u_{3}(n t):=\frac{1-f_{n t, \tau(n t, n)}(s)}{e^{S_{\tau(n t, n)}-S_{n t}}} ; & u_{4}(n t):=f_{\tau(n t, n), n}(0)
\end{array}
$$

и $\mathbf{u}(n)=\mathbf{u}(n, t):=\left(u_{1}(n t), u_{2}(n t), u_{3}(n t), u_{4}(n t)\right)$. Вспоминая определения и свойства (2)-(4), введем четырехмерный вектор

$$
\mathbf{u}(\infty):=\left(\zeta^{-}(s), q^{+}, \zeta^{*-}(s), q^{*+}\right)
$$

компоненты которого независимы, причем $\zeta^{*-}(s) \stackrel{d}{=} \zeta^{-}(s), q^{*+} \stackrel{d}{=} q^{+}$. Таким образом, для соответствующих множеств $\mathscr{C}_{i}, i=1,2,3,4$,

$$
\begin{aligned}
\mathbf{P} & \left\{\left(\zeta^{-}(s), q^{+}, \zeta^{*-}(s), q^{*+}\right\} \in\left(\mathscr{C}_{1}, \mathscr{C}_{2}, \mathscr{C}_{3}, \mathscr{C}_{4}\right)\right) \\
& =\widehat{\mathbf{P}}^{-}\left(\zeta^{-}(s) \in \mathscr{C}_{1}\right) \widehat{\mathbf{P}}^{+}\left(q^{+} \in \mathscr{C}_{2}\right) \widehat{\mathbf{P}}^{-}\left(\zeta^{-}(s) \in \mathscr{C}_{3}\right) \widehat{\mathbf{P}}^{+}\left(q^{+} \in \mathscr{C}_{4}\right) .
\end{aligned}
$$

Лемма 9. Если выполнень условия $A 2-A 3$, то для любого $t \in(0,1)$

$$
\{\mathbf{u}(n) \mid \tau(n)<n t\} \stackrel{w}{\longrightarrow} \mathbf{u}(\infty), \quad n \rightarrow \infty .
$$

Д ок аз а т ель с т в о. Ясно, что для $0 \leqslant k_{1}<n t \leqslant k_{2} \leqslant n$

$$
\begin{aligned}
\mathscr{B}\left(k_{1}, k_{2}, t, n\right):= & \left\{\min _{0 \leqslant j<n t} S_{j} \leqslant \min _{n t \leqslant j \leqslant n} S_{j}\right\} \\
& \bigcap\left\{\min _{0 \leqslant l<k_{1}} S_{l}>S_{k_{1}}, \min _{k_{1} \leqslant l<n t} S_{l} \geqslant S_{k_{1}}\right\} \\
& \bigcap\left\{\min _{0 \leqslant l<k_{2}} S_{l}>S_{k_{2}}, \min _{k_{2} \leqslant l<n t} S_{l} \geqslant S_{k_{2}}\right\} \\
= & \left\{\tau(n)=k_{1}, \tau(n t, n)=k_{2}\right\} .
\end{aligned}
$$

Полагая $\boldsymbol{\lambda}=\left(\lambda_{1}, \lambda_{2}, \lambda_{3}, \lambda_{4}\right)$, имеем

$$
\begin{aligned}
\mathbf{E} & {[\exp \{-(\boldsymbol{\lambda}, \mathbf{u}(n))\} ; \tau(n)<n t] } \\
& =\sum_{0 \leqslant k_{1}<n t} \sum_{n t \leqslant k_{2} \leqslant n} \mathbf{E}\left[\exp \{-(\boldsymbol{\lambda}, \mathbf{u}(n))\} ; \mathscr{B}\left(k_{1}, k_{2}, t, n\right)\right] .
\end{aligned}
$$

Заметим, что, согласно обобщенному закону арксинуса, вклад в двойную сумму всех слагаемых, взятых по множеству

$$
\begin{aligned}
\mathscr{C}_{\varepsilon}(n):= & \left\{0 \leqslant k_{1}<n t \varepsilon\right\} \bigcup\left\{n t(1-\varepsilon) \leqslant k_{1}<n t\right\} \\
& \bigcup\left\{n t \leqslant k_{2}<n t(1+\varepsilon)\right\} \bigcup\left\{n(1-\varepsilon) \leqslant k_{2} \leqslant n\right\},
\end{aligned}
$$

стремится к нулю, если сначала $n \rightarrow \infty$, а затем $\varepsilon \rightarrow 0$.

Рассмотрим четыре независимых реализации случайной среды $\left(f_{k}^{(i)}\right)_{k \geqslant 0}, i=1,2,3,4$, соответствующие им независимые сопровождающие случайные блуждания $\left(S_{k}^{(i)}\right)_{k \geqslant 0}, i=1,2,3,4$, вектор

$$
\mathbf{u}^{*}(n)=\mathbf{u}^{*}\left(n, t, k_{1}, k_{2}\right):=\left(u_{1}^{*}\left(k_{1}\right), u_{2}^{*}\left(n t-k_{1}\right), u_{3}^{*}\left(k_{2}-n t\right), u_{4}^{*}\left(n-k_{2}\right)\right)
$$


с независимыми компонентами

$$
\begin{array}{rlrl}
u_{1}^{*}\left(k_{1}\right) & :=\frac{1-f_{k_{1}, 0}^{(1)}(s)}{e^{S_{k_{1}}^{(1)}}}=\zeta_{k_{1}}^{-}(s), & u_{2}^{*}\left(n t-k_{1}\right):=f_{0, n t-k_{1}}^{(2)}(0), \\
u_{3}^{*}\left(k_{2}-n t\right):=\frac{1-f_{k_{2}-n t, 0}^{(3)}(s)}{e^{S_{k_{2}-n t}^{(3)}}}:=\zeta_{k_{2}-n t}^{*-}(s), & u_{4}^{*}\left(n-k_{2}\right):=f_{0, n-k_{2}}^{(4)}(0)
\end{array}
$$

и множество

$$
\begin{aligned}
\mathscr{B}^{*}\left(k_{1}, k_{2}, t, n\right):= & \left\{S_{n t-k_{1}}^{(2)} \geqslant-S_{k_{2}-n t}^{(3)}\right\} \\
& \cap\left\{\max _{1 \leqslant j \leqslant k_{1}} S_{j}^{(1)}<0, \min _{0 \leqslant j<n t-k_{1}} S_{j}^{(2)} \geqslant 0\right\} \\
& \cap\left\{\max _{1 \leqslant j \leqslant k_{2}-n t} S_{j}^{(3)}<0, \min _{0 \leqslant j<n-k_{2}} S_{j}^{(4)} \geqslant 0\right\} .
\end{aligned}
$$

Используя принцип двойственности, несложно проверить, что

$\mathbf{E}\left[\exp \{-(\boldsymbol{\lambda}, \mathbf{u}(n))\} ; \mathscr{B}\left(k_{1}, k_{2}, t, n\right)\right]=\mathbf{E}\left[\exp \left\{-\left(\boldsymbol{\lambda}, \mathbf{u}^{*}(n)\right)\right\} ; \mathscr{B}^{*}\left(k_{1}, k_{2}, t, n\right)\right]$.

Обозначим $\Gamma^{(i)}:=\min \left\{n \geqslant 1: S_{n}^{(i)} \geqslant 0\right\}, \gamma^{(i)}=\min \left\{n \geqslant 1: S_{n}^{(i)}<0\right\}$ и положим

$$
T_{n t-k_{1}, k_{2}-n t}:=\exp \left\{-\lambda_{2} u_{2}^{*}\left(n t-k_{1}\right)-\lambda_{3} u_{3}^{*}\left(k_{2}-n t\right)\right\}
$$

и

$\mathscr{B}_{1}^{*}\left(k_{1}, k_{2}, t, n\right):=\left\{S_{n t-k_{1}}^{(2)} \geqslant-S_{k_{2}-n t}^{(3)}\right\} \cap\left\{\gamma^{(2)}>n t-k_{1}\right\} \cap\left\{\Gamma^{(3)}>k_{2}-n t\right\}$.

В силу независимости рассматриваемых случайных блужданий, имеем

$$
\begin{aligned}
\mathbf{E}\left[\exp \left\{-\left(\boldsymbol{\lambda}, \overline{\mathbf{u}}^{*}(n)\right)\right\} ; \mathscr{B}^{*}\left(k_{1}, k_{2}, t, n\right)\right] \\
=\mathbf{E}\left[\exp \left\{-\lambda_{1} u_{1}^{*}\left(k_{1}\right)\right\} \mid \Gamma^{(1)}>k_{1}\right] \mathbf{E}\left[T_{n t-k_{1}, k_{2}-n t} \mid \mathscr{B}_{1}^{*}\left(k_{1}, k_{2}, t, n\right)\right] \\
\quad \times \mathbf{E}\left[\exp \left\{-\lambda_{4} u_{4}^{*}\left(n-k_{2}\right)\right\} \mid \gamma^{(4)}>n-k_{2}\right] \mathbf{P}\left(\mathscr{B}^{*}\left(k_{1}, k_{2}, t, n\right)\right) .
\end{aligned}
$$

Мы знаем (см. (2)-(4)), что $\lim _{k \rightarrow \infty} u_{i}^{*}(k)=\zeta^{-}(s) \widehat{\mathbf{P}}^{-}$-п.н. для $i=1,3$ и $\lim _{k \rightarrow \infty} u_{i}^{*}(k)=q^{+} \widehat{\mathbf{P}}^{+}$-п.н. для $i=2,4$. В частности, это означает, что $\widehat{\mathbf{P}}$-п.н. сушествует предел

$$
T:=\lim _{\min \left(n t-k_{1}, k_{2}-n t\right) \rightarrow \infty} T_{n t-k_{1}, k_{2}-n t}=e^{-\lambda_{2} q^{+}-\lambda_{3} \zeta^{-}(s)},
$$

и по лемме 8

$$
\mathbf{E}\left[T_{n t-k_{1}, k_{2}-n t} \mid \mathscr{B}_{1}^{*}\left(k_{1}, k_{2}, t, n\right)\right] \longrightarrow \widehat{\mathbf{E}}\left[e^{-\lambda_{2} q^{+}-\lambda_{3} \zeta^{-}(s)}\right], \quad n \rightarrow \infty,
$$

равномерно по

$\left(k_{1}, k_{2}\right) \in \mathscr{C}_{\varepsilon}^{*}(n):=\left\{\left(l_{1}, l_{2}\right): l_{1} \in[n t \varepsilon, n t(1-\varepsilon)], l_{2} \in[n t(1+\varepsilon), n(1-\varepsilon)]\right\}$. 
Аналогично, в силу леммы 3 статьи [10]

$$
\begin{gathered}
\lim _{k_{1} \rightarrow \infty} \mathbf{E}\left[\exp \left\{-\lambda_{1} u_{1}^{*}\left(k_{1}\right)\right\} \mid \Gamma^{(1)}>k_{1}\right]=\widehat{\mathbf{E}}^{-}\left[\exp \left\{-\lambda_{1} \zeta^{-}(s)\right\}\right], \\
\lim _{n-k_{2} \rightarrow \infty} \mathbf{E}\left[\exp \left\{-\lambda_{4} u_{1}^{*}\left(n-k_{2}\right)\right\} \mid \gamma^{(4)}>n-k_{2}\right]=\widehat{\mathbf{E}}^{+}\left[\exp \left\{-\lambda_{4} q^{+}\right\}\right] .
\end{gathered}
$$

Следовательно, при $n \rightarrow \infty$

$$
\begin{aligned}
& \mathbf{E}\left[\exp \left\{-\left(\boldsymbol{\lambda}, \overline{\mathbf{u}}^{*}(n)\right)\right\} \mid \mathscr{B}^{*}\left(k_{1}, k_{2}, t, n\right)\right] \\
& \quad \longrightarrow(\widehat{\mathbf{E}} \times \widehat{\mathbf{E}})\left[\exp \left\{-\lambda_{1} \zeta^{-}(s)-\lambda_{2} q^{+}-\lambda_{3} \zeta^{*-}(s)-\lambda_{4} q^{*+}\right\}\right] \\
& \quad=\mathbf{E}[\exp \{-(\boldsymbol{\lambda}, \overline{\mathbf{u}}(\infty))\}]
\end{aligned}
$$

равномерно по $\left(k_{1}, k_{2}\right) \in \mathscr{C}_{\varepsilon}^{*}(n)$.

Таким образом, для любого $\delta>0$ и всех достаточно больших $n$ мы получаем

$$
\begin{aligned}
\mathbf{E} & {[\exp \{-(\boldsymbol{\lambda}, \overline{\mathbf{u}}(n))\} ; \tau(n)<n t] } \\
& =\sum_{0 \leqslant k_{1}<n t} \sum_{n t \leqslant k_{2} \leqslant n} \mathbf{E}\left[\exp \left\{-\left(\boldsymbol{\lambda}, \overline{\mathbf{u}}^{*}(n)\right)\right\} ; \mathscr{B}^{*}\left(k_{1}, k_{2}, t, n\right)\right] \\
& \leqslant \mathbf{P}\left(\mathscr{C}_{\varepsilon}(n)\right)+(1+\delta) \mathbf{E}[\exp \{-(\boldsymbol{\lambda}, \overline{\mathbf{u}}(\infty))\}] \sum_{\left(k_{1}, k_{2}\right) \in \mathscr{C}_{\varepsilon}^{*}(n)} \mathbf{P}\left\{\mathscr{B}^{*}\left(k_{1}, k_{2}, t, n\right)\right\} \\
& =\mathbf{P}\left(\mathscr{C}_{\varepsilon}(n)\right)+(1+\delta) \mathbf{E}[\exp \{-(\boldsymbol{\lambda}, \overline{\mathbf{u}}(\infty))\}] \mathbf{P}\left((\tau(n), \tau(n t, n)) \in \mathscr{C}_{\varepsilon}^{*}(n)\right) .
\end{aligned}
$$

При помощи таких же рассуждений нетрудно получить аналогичную оценку снизу. Отсюда легко следует утверждение леммы, поскольку

$$
\lim _{\varepsilon \downarrow \rightarrow 0} \lim _{n \rightarrow \infty} \mathbf{P}\left((\tau(n), \tau(n t, n)) \in \mathscr{C}_{\varepsilon}^{*}(n)\right)=1
$$

и величина $\delta$ может быть выбрана сколь угодно малой.

Доказательство те о ремы 9. Пусть

$$
L(t, n):=\frac{e^{S_{\tau(n t, n)}-S_{n t}}\left(1-f_{0, n t}(0)\right)}{e^{S_{\tau(n t)}}\left(1-f_{n t, n}(0)\right)} .
$$

Непосредственные вычисления дают

$$
\alpha_{n}(n t):=e^{S_{\tau(n t, n)}-S_{\tau(n)}} \frac{\beta_{n}(n t)}{L(t, n)}=\frac{1-f_{0, n}(0)}{1-f_{0, n t}(0)} .
$$

Согласно следствию 3 работы [10] для любого $\varepsilon>0$

$$
\lim _{n \rightarrow \infty} \mathbf{P}\left\{\left|\alpha_{n}(n t)-1\right|>\varepsilon \mid \tau(n)<n t\right\}=0 .
$$

Следовательно, для завершения доказательства теоремы достаточно показать, что при $n \rightarrow \infty$

$$
\{L(t, n) \mid \tau(n)<n t\} \stackrel{w}{\longrightarrow} \frac{\zeta^{-}\left(q^{+}\right)}{\zeta^{*-}\left(q^{*}\right)} .
$$

Но этот факт является очевидным следствием леммы 9 .

Теорема доказана. 


\section{СПИСОК ЛИТЕРАТУРЫ}

1. Athreya K. B., Karlin S. On branching processes with random environments. I. Extinction probabilities. - Ann. Math. Statist., 1971, v. 42, № 5, p. 1499-1520.

2. Athreya K. B., Karlin $S$. On branching processes with random environments. II. Limit theorems. - Ann. Math. Statist., 1971, v. 42, № 6, p. 1843-1858.

3. Athreya K. B., Ney P.E. Branching Processes. New York-Heidelberg: SpringerVerlag, 1972, $287 \mathrm{p}$.

4. Afanasyev V.I., Geiger J., Kersting G., Vatutin V.A. Criticality for branching processes in random environment. - Ann. Probab., 2005, v. 33, № 2, p. 645-673.

5. Borovkov K. A., Vatutin V.A. Reduced critical branching processes in random environment. - Stochastic Process. Appl., 1997, v. 71, № 2, p. 225-240.

6. Ватутин В.A. Редуцированные ветвящиеся процессы в случайной среде: критический случай. - Теория вероятн. и ее. примен., 2002, т. 47, в. 1, с. 21-38.

7. Vatutin V.A., Dyakonova E.E. Reduced branching processes in random environment. - Mathematics and Computer Science. II: Algorithms, Trees, Combinatorics and Probabilities. Ed. by B. Chauvin et al. Basel: Birkhäuser, 2002, p. 455-467.

8. Vatutin V.A., Dyakonova E. E. Yaglom type limit theorem for branching processes in random environment. - Mathematics and Computer Science. III: Algorithms, Trees, Combinatorics and Probabilities. Ed. by M. Drmota et al. Basel: Birkhäuser, 2004, p. 375-385.

9. Ватутин В. А., Дьяконова E. Е. Ветвящиеся процессы Гальтона-Ватсона в случайной среде. I: Предельные теоремы. - Теория вероятн. и ее. примен., 2003, т. 48 , в. 2 , с. $274-300$.

10. Ватутин В. А., Дьяконова Е. Е. Ветвящиеся процессы Гальтона-Ватсона в случайной среде. II: конечномерные распределения. - Теория вероятн. и ее. примен., 2004 , т. 49 , в. 2 , с. $231-268$.

11. Ватутин B.A., Дьяконова E. E. Ветвящиеся процессы в случайной среде и бутылочные горлышки в эволюции популяций. - Теория вероятн. и ее. примен., 2006 , т. 51 , в. 1 , с. $22-46$.

12. Geiger J., Kersting G. The survival probability of a critical branching process in random environment. - Теория вероятн. и ее примен., 2000, т. 45, в. 3, с. 607-615.

13. Doney $R$. A. Spitzer's condition and the ladder variables in random walks. - Probab. Theory Related Fields, 1995, v. 101, № 4, p. 577-580.

14. Durrett $R$. Conditioned limit theorems for some null recurrent Markov processes. Ann. Probab., 1978, v. 6, № 5, p. 798-828.

15. Зубков A.M. Предельные распределения расстояния до ближайшего общего предка. - Теория вероятн. и ее примен., 1975, т. 20, в. 3, с. 614-623.

16. Спицер $\Phi$. Принципы случайного блуждания. М.: Мир, 1969, 472 с.

17. Fleischmann K., Vatutin V. A. Reduced subcritical branching processes in a random environment. - Adv. Appl. Probab., 1999, v. 31, № 1, p. 88-111.

18. Fleischmann K., Prehn U. Ein Grenzwertsatz für subkritische Verzweigungsprozesse mit endlich vielen Typen von Teilchen. - Math. Nachr., 1974, v. 64, p. 357-362.

19. Fleischmann K., Siegmund-Schultze R. The structure of reduced critical GaltonWatson processes. - Math. Nachr., 1977, v. 79, p. 233-241.

Поступила в редакцию 27.III.2006 